Revue d'histoire de l'Amérique française

BRS REVUE D.HISTOIRE DE L'AMÉRIQUE FRANÇAISE

\title{
La liste civile du Bas-Canada (1794-1812) : Un essai d'économie historique (suite)
}

\section{Gilles Paquet et Jean-Pierre Wallot}

Volume 24, numéro 1, juin 1970

URI : https://id.erudit.org/iderudit/302954ar

DOI : https://doi.org/10.7202/302954ar

Aller au sommaire du numéro

Éditeur(s)

Institut d'histoire de l'Amérique française

ISSN

0035-2357 (imprimé)

1492-1383 (numérique)

Découvrir la revue

Citer cet article

Paquet, G. \& Wallot, J.-P. (1970). La liste civile du Bas-Canada (1794-1812) : Un essai d'économie historique (suite). Revue d'histoire de l'Amérique française,

24(1), 3-43. https://doi.org/10.7202/302954ar d'utilisation que vous pouvez consulter en ligne.

https://apropos.erudit.org/fr/usagers/politique-dutilisation/ 


\section{LA LISTE CIVILE DU BAS-CANADA (1794-1812): UN ESSAI D'ÉCONOMIE HISTORIQUE

\author{
(suite) *
}

\section{La liste civile: une analyse quantitative}

Les débats sur les emplois publics et la liste civile réfléchissent une partie de la réalité. Mais jusqu'à quel point les faits coïncident-ils avec la perception que s'en font les différents groupes en présence. Ainsi, le parti canadien inculpe sans appel les Britanniques et, à un moindre degré, les "chouayens", i.e. les Canadiens qui se seraient assimilés, de rafler presque toutes les places. Mais dans la section IV, nous avons entrevu les démarches nombreuses et souvent inutiles de l'ancienne élite. Cette régularité monotone des revendications de la part des Canadiens étonne d'ailleurs un observateur britannique: “... they expect a line of conduct from the English, that the English would not experience from them, were situations changed; but the fact is, they hold a large share of the public employments." ${ }^{152}$ Par ailleurs, certains nobles canadiens, qui profitent du régime, semblent plutôt satisfaits. Ainsi, Chartier de Lotbinière, conseiller législatif et exécutif, écrit à un ami que le Chevalier de Niverville et son frère Montizambert, tous deux très âgés, vivent "des bienfaits du gouvernement lequel est très généreux et sous lequel les Canadiens sont très heureux en général". ${ }^{153}$ Les témoignages, que nous pourrions multiplier et contraster à loisir, s'avèrent donc jusqu'à un certain point

* Voir notre Revue, 23 (1969-1970): 209-230; 361-392. Errata: à la page 384, au 2e paragraphe, à la 6e ligne, il fallait lire £51 627 et non $£ 5627$; p. 391, 3e ligne du bas, 1811 et non 1805 .

152 Hugh Gray, Letters from Canada Written during a Residence there in the Years 1806, 1807, and 1808 [...] (Londres, 1809), 331.

153 Eustache Gaspard Alain Chartier de Lotbinière, seigneur de Vaudreuil, au comte des Méloizes (en France), Vaudreuil, 8 septembre 1802, $A P Q$, gr. coll., lettres au comte des Méloizes (copies). Le même seigneur félicite François Baby, un autre favori du régime, d'avoir "été gratifié d'une pension de $\$ 150$ réversible sur la tête de votre respectable épouse... Je suis vraiment heureux de savoir que vos services et votre mérite soient récompensés et très satisfait que la générosité de Notre bon Maître s'étende au-delà de votre existence." (Le même à l'hon. F. Baby, Vaudreuil, 26 septembre 1802, APQ, gr. coll., papiers P.-G. Roy (copies), 15: 166). 
contradictoires. Il convient donc d'y regarder de plus près. La présente section retrace l'évolution quantitative de la liste civile en général, c'est-à-dire des emplois publics et des pensions. Elle tente aussi, dans la mesure du possible, d'en profiler les composantes sociale, ethnique et régionale.

\section{(1) Les données}

L'étude quantitative qui suit porte exclusivement sur les salaires et les pensions de la liste civile officielle, ainsi que sur quelques salaires d'emplois spécifiques (une douzaine en 1812, encore moins auparavant) pourvus par des lois spéciales de l'Assemblée (service législatif, pilotage sur le fleuve SaintLaurent), entre 1794 et $1812 .{ }^{154}$ Les données proviennent des Journaux de la Chambre d'Assemblée du Bas-Canada. Elles ne comprennent donc pas les pensions et les salaires secrets, non plus que les emplois militaires - hormis ceux de la milice, qui dépendent de lois de l'Assemblée. Comme certains salaires et pensions sont inscrits en livres "courant" (i.e. cours d'Halifax et de Québec), mais la grande majorité, en livres "sterling", nous avons transposé les premiers en livres sterling selon un taux de change de 10/9.155 Dans nos calculs, nous avons ignoré les fractions de pence (v.g. £5.3.111/2 devient £5.3.11) et les rares contrats insérés dans la liste civile (surtout des loyers et des frais d'impression pour des publications de la législature) : ceux-ci ne constituent qu'une infime proportion des contrats de l'Etat, la plupart étant défrayés par des lois particulières et surtout par la caisse militaire; il n'est d'ailleurs pas possible d'y disjoindre la portion des salaires de celle des autres coûts. Selon la même logique, nous avons encore retranché les simples remboursements de dépenses (frais de voyage, allocations de subsistance, etc.), qui ne comportent aucune rémunération et auraient soufflé exagérément la part des dépenses civiles allant aux Britanniques. De même, nous avons biffé les sommes allouées aux officiers rapporteurs, en années d'élections, car il s'agit de simples remboursements de frais jusqu'en 1809 et de montants généralement inférieurs à $£ 10$ st. Leur inclusion eût en outre gauchi le nombre de places en faveur des Canadiens.

Quelques rares allocations allient des proportions indéterminées de dépenses et d'honoraires, ceux-ci semblant plus

154 En 1793, la liste civile est trop minime pour servir de point de départ utile.

155 C'est-à-dire de 10 livres courant pour 9 livres sterling. C'est l'équivalence utilisée généralement à l'époque, bien que la valeur exacte de l'échange soit la suivante: $£ 111.2 .3 \mathrm{c}$. pour $£ 100$ st. 
importants que celles-là. Comme nous n'avons pu dissocier les deux composantes et qu'il s'agit tout au plus de trois cas dans chacune des grosses années, impliquant des dépenses totales annuelles de l'ordre de $£ 500$ à $£ 1000$ st. au maximum, nous les avons comptées comme des rémunérations.

Pour chaque salaire et pension, nous avons cueilli des renseignements qui nous permettent de repérer le titulaire selon des coordonnées géographiques, ethniques et économiques:

I. - Localisation géographique:

Québec

Montréal

Trois-Rivières

Gaspé

localisation multiple dans le Bas-Canada 156

localisation hors du Bas-Canada

région inconnue

II. - Ethnie:

Canadiens français

Canadiens anglais ou Britanniques

statut ethnique inconnu

III. - Grandeur exacte du salaire ou de la pension.

Grâce à une codification fort simple, nous avons compilé de telles données à propos de chaque salaire et de chaque pension selon une forme strictement numérique, pour les transcrire ensuite sur des cartes perforées. L'analyse de ces informations statistiques, emmagasinées ainsi de facon fort compacte, s'est faite au moyen de procédés mécanographiques.

$\mathrm{Au}$ premier abord, on peut croire qu'une exactitude très grande caractérise ces manipulations des données. En fait, la qualité des intrants circonscrit évidemment la valeur des résultats d'une telle analyse. Or la précision ne saurait être absolue au niveau de ces intrants. Outre la possibilité toujours présente d'erreurs de transcription et de codification, malgré une triple vérification de chaque observation, certaines difficultés spécifiques émanent de la nature même du matériau historique. Ainsi, à cause de sérieuses lacunes dans les dictionnaires biographiques et autres instruments de travail, il a pu nous arriver de classifier un fonctionnaire ou un pensionné dans la mauvaise ethnie ou la mauvaise région. Certains noms, britanniques en apparence, sont français: il est plus difficile de savoir que "Lewis" Montizambert

156 Certains fonctionnaires gagnent un salaire en voyageant régulièrement à travers les différents districts de la province. 
est canadien-français que dans le cas de "Peter" Panet. D'autres noms d'origine européenne (surtout suisse) semblent au contraire canadiens-français et sont britanniques (v.g. William Bouthillier). Mais ces cas-limites, très rares, se trouvent surtout dans les pensions et exclusivement dans les paliers inférieurs de salaires et de pensions. En effet, on connaît très bien les hauts fonctionnaires. Lorsqu'il y a doute, par exemple dans le cas des régions (surtout pour les pensions, alors que souvent la liste civile ne reproduit même pas les initiales des pensionnés), nous avons toujours utilisé le code "inconnu".

Quelques erreurs encore possibles, réparties sur l'ensemble des années et limitées aux catégories inférieures, ne sauraient vraiment modifier la configuration globale décelée par nos analyses ni infirmer la valeur de nos résultats. Cependant, pour nous prémunir contre une telle éventualité, nous avons eu recours à un instrument de contrôle: une compilation antérieure faite plus rapidement et sans le recours aux procédés mécanographiques. ${ }^{157}$ Tout écart significatif eût entraîné une vérification additionnelle, le cas échéant. Dans le détail, on peut détecter certaines variations annuelles qui sont dues au décès d'un ou de plusieurs fonctionnaires: leurs noms et ceux de leurs remplaçants sont inscrits dans la liste civile, avec leur fraction respective de la rémunération attachée à la même ou aux mêmes fonctions. Il faut tenir compte aussi de la création de nouveaux postes, de l'arrêt de certains cumuls, etc. Mais dans l'ensemble, si l'on ajuste les données pour en extraire l'effet net de la croissance annuelle de la fonction publique, les chiffres sont relativement stables.

Dernier point à noter, dans le secteur des salaires, nos données renvoient à des fonctions et non à des individus. En fait, les cumuls de fonctions amenuisent le nombre réel de fonctionnaires jusqu'à un total bien inférieur aux chiffres cités. A cause du coût élevé d'un projet qui eût englobé toutes les informations pertinentes, y compris les noms des titulaires, nous nous sommes contentés de relever les cumuls en examinant attentivement la liste civile. Toute erreur, ici, et il $y$ en a sûrement, ne peut qu'être d'omission: nous avons consigné tous les cumuls que nous avons repérés, mais certains ont pu passer inaperçus. Une tendance intéressante se dessine dans l'importance relative des cumuls (tableau VI) : alors que chez les Canadiens anglais, ils se situent à environ $20 \%$ des fonctions tout

157 G. Paquet et J.-P. Wallot, "Lower Canada, 1792-1812 [...]", tableaux 13 et 14 . 
au long de ces années, avec des oscillations entre 15 et $25 \%$, pour les Canadiens français, ce pourcentage, autour de $10 \%$ (en fait entre 3 et $13 \%$ ) entre 1794 et 1802, se déplace brusquement jusqu'aux environs de $20 \%$ pour le reste de la période. Le cumul des fonctions, qui est deux fois plus important chez les Canadiens anglais que chez les Canadiens français durant la première moitié de la période, joue en proportion égale pour l'un et l'autre groupes dans la seconde moitié.

TABLEAU VI

Importance relative des cumuls de fonctions (1794-1812)

\begin{tabular}{|c|c|c|c|c|c|c|c|}
\hline & \multicolumn{2}{|c|}{ Nombre de salaires } & \multicolumn{2}{|c|}{$\begin{array}{c}\text { Nombre de } \\
\text { postes cumulés * }\end{array}$} & \multicolumn{2}{|c|}{$\frac{(3)}{(1)} \begin{array}{c}\text { Propor- } \\
\text { tion }\end{array}$} & \multirow{2}{*}{$\frac{(4)}{(2)}$} \\
\hline & (1) & (2) & (3) & (4) & (5) & & \\
\hline & CF & CA & CF & $\mathrm{CA}$ & $\begin{array}{l}\text { CF } \\
\%\end{array}$ & & $\underset{\%}{\mathrm{CA}}$ \\
\hline 1794 & 30 & 78 & 4 & 8 & 13.3 & & 10.3 \\
\hline & 30 & 77 & 3 & 14 & 10.0 & & 18.2 \\
\hline 6 & 30 & 79 & 4 & 15 & 13.3 & & 19.0 \\
\hline 7 & 32 & 82 & 3 & 16 & 9.4 & & 19.5 \\
\hline 8 & 31 & 79 & 1 & 16 & 3.2 & & 20.2 \\
\hline & 32 & 83 & 1 & 17 & 3.1 & & 20.5 \\
\hline 1800 & 36 & 87 & 4 & 16 & 11.1 & & 18.4 \\
\hline 1 & 33 & 87 & 3 & 21 & 9.1 & & 24.1 \\
\hline 2 & 35 & 88 & 5 & 20 & 14.3 & & 22.7 \\
\hline 3 & 38 & 90 & 9 & 14 & 23.7 & & 15.6 \\
\hline 4 & 48 & 83 & 11 & 14 & 22.9 & & 16.9 \\
\hline 5 & 52 & 99 & 9 & 19 & 17.3 & & 19.2 \\
\hline 6 & 56 & 99 & 12 & 18 & 21.4 & & 18.2 \\
\hline 7 & 58 & 110 & 14 & 28 & 24.1 & & 25.4 \\
\hline 8 & 71 & 115 & 12 & 24 & 16.9 & & 20.9 \\
\hline 9 & 61 & 110 & 14 & 18 & 22.9 & & 16.4 \\
\hline 1810 & 70 & 109 & 16 & 18 & 22.9 & & 16.5 \\
\hline 1 & 75 & 120 & 18 & 22 & 24.0 & & 18.3 \\
\hline 2 & 79 & 130 & 18 & 26 & 22.8 & & 20.0 \\
\hline
\end{tabular}

Source: $J C A B C, 1794-1813$.

* Nous avons agrégé les cumuls de fonctions sans chercher à les analyser par le détail en diverses catégories: cumuls simples (lorsqu'une personne occupe 2 emplois), cumuls doubles ( 3 emplois), cumuls triples (4 emplois), etc. Toutefois, nous avons, bien sûr, tenu compte des cumuls doubles, triples, etc. dans nos calculs. Ainsi, en 1794, pour les Canadiens français, les 4 cumuls qui figurent dans la colonne (3) comprennent en fait 2 cumuls simples et 1 cumul double, soit 4 postes cumulés. Pour les Canadiens anglais, il s'agit de 5 cumuls simples et de 1 cumul triple, soit 8 postes cumulés. 
D'autre part, le bond dans le nombre de postes détenus par des Canadiens français, après 1803, s'accompagne d'un saut correspondant dans le nombre de postes cumulés (voir tableau VI). On pourrait donc croire que la montée dans le nombre de postes ne correspond pas à une hausse du même ordre dans le nombre d'individus. Une comparaison de la croissance des uns et des autres (voir tableau VII), dans le groupe canadienfrançais, montre bien que l'évolution du nombre de postes et celle du nombre d'individus suivent des sentiers parallèles. On peut donc utiliser la structure chronologique des postes comme un indicateur sûr de celle des individus impliqués.

(2) Un court excursus sur les classes sociales

Outre qu'il n'a pas été possible de tenir une arithmétique précise des cumuls, nous n'avons pu scruter et démonter la structure des classes sociales qui définit le contingent des

\section{TABLEAU VII}

Structure ethnique des postes et des individus

$$
\text { (1794-1812) }
$$

$\begin{array}{cccccc}\text { total } & \text { total } & \% & \text { total } & & \\ \text { des postes } & \text { des postes } & \begin{array}{c}\text { des } \\ \text { total }\end{array} & \% \\ \text { des postes } & \text { CF } & \text { CF } & \text { individus } & \text { des CF } & \text { des CF } \\ \text { (1) } & (2) & (3) & (4) & (5) & (6)\end{array}$

$\begin{array}{rrrrrrr}1794 & 108 & 30 & 27.8 & 96 & 26 & 27.1 \\ 5 & 107 & 30 & 28.0 & 90 & 27 & 30.0 \\ \mathbf{6} & 109 & 30 & 27.5 & 90 & 26 & 28.9 \\ 7 & 114 & 32 & 28.1 & 95 & 29 & 30.5 \\ 8 & 110 & 31 & 28.2 & 93 & 30 & 32.3 \\ 9 & 115 & 32 & 27.8 & 97 & 31 & 32.0 \\ 1800 & 123 & 36 & 29.3 & 103 & 32 & 31.1 \\ 1 & 120 & 33 & 27.5 & 96 & 30 & 31.2 \\ 2 & 123 & 35 & 28.5 & 98 & 30 & 30.6 \\ 3 & 128 & 38 & 29.7 & 105 & 29 & 27.6 \\ 4 & 131 & 48 & 36.6 & 106 & 37 & 34.9 \\ 5 & 151 & 32 & 34.4 & 123 & 43 & 35.0 \\ 6 & 155 & 56 & 36.1 & 125 & 44 & 35.2 \\ 7 & 168 & 58 & 34.5 & 126 & 44 & 34.9 \\ 8 & 186 & 71 & 38.2 & 150 & 59 & 39.3 \\ 9 & 171 & 61 & 35.7 & 139 & 47 & 33.8 \\ 1810 & 179 & 70 & 39.1 & 145 & 54 & 37.2 \\ 1 & 195 & 75 & 38.5 & 155 & 57 & 36.8 \\ 2 & 209 & 79 & 37.8 & 165 & 61 & 37.0\end{array}$

Source: Tableau VI. 
“gens à places”. L'absence de données biographiques suffisantes, les imprécisions et les erreurs dans la liste civile elle-même, la difficulté enfin de nous fier aux seuls noms pour déterminer l'origine sociale: autant de raisons qui nous ont fait renoncer au projet initial d'une sociographie des détenteurs de postes.

Cependant, du côté canadien-français, il nous a paru possible et utile de dresser un inventaire grossier et partiel qui nous démasque quand même certains phénomènes. Nous reproduisons cet exercice dans le tableau VIII. Tout en délimitant le champ un peu étroit de notre exploration, ces balises découvrent une autre dimension des données que nous n'avons pu encore vraiment exploiter.

Le tableau VIII répertorie une partie des Canadiens français qui détiennent des postes rapportant $£ 10$ st. ou plus, ou qui reçoivent une pension, en quelques catégories sociales découpées à gros traits: les "nobles" $(\mathrm{N})$, y compris des professionnels (P), les "non-nobles" (NN) ou les autres, avec ici encore le nombre de professionnels (P). Par conséquent, cette classification ne comprend pas les individus dont nous n'avons pu préciser l'origine sociale. ${ }^{158}$

L'historiographie affirme que la liste civile privilégiait la noblesse. ${ }^{159}$ De fait, une lecture rapide nous $\mathrm{y}$ fait repérer les

158 Nous avons pu identifier socialement environ $75 \%$ des effectifs au niveau des postes et de 50 à $75 \%$ d'entre eux au niveau des pensions. D'autre part, nous aurions naturellement préféré une grille sociale moins grossière et une ventilation plus détaillée des occupations. Mais pour les raisons évoquées au début de cet excursus, nous n'avons pas voulu nous hasarder dans une distribution plus fine (nobles, bourgeois, artisans, etc.). Toutefois, une connaissance approfondie de la période et d'un grand nombre d'individus, les outils de travail disponibles et quelques rares précisions dans la liste civile nous ont permis une classification sommaire de la majorité des fonctionnaires et des pensionnés. En ce qui a trait aux professionnels, i.e. aux avocats, notaires et arpenteurs (il n'y a pas de médecins canadiensfrançais dans la liste civile), leur rôle plus important et les listes dans l'Almanach de Québec ont rendu possible une identification plus certaine. Par "nobles" $(N)$, nous entendons la noblesse seigneuriale et militaire, $\mathrm{y}$ compris ceux de ses membres qui sont aussi professionnels (v.g. de Bonne, J.-A. Panet). Les personnes d'origine "non-noble" (NN) comprennent les autres, dont des professionnels.

159 Ainsi qu'une "clique" de Britanniques. Voir, par exemple, Robert Christie, History of the Late Province of Lower Canada [...] (6 vol., Québec, 1848), 1: 347 sqq.; W. Kingsford, The History of Canada (10 vol., Toronto, 1887-1898), 9: 507 sqq.; A. R. M. Lower, Canadians in the Making. A Social History of Canada (Toronto, 1958), 128 sqq.; H. T. Manning, The Revolt of French Canada (1800-1835) [...] (Toronto, 1962), 78 sqq.; T. Chapais, Cours d'histoire du Canada (8 vol., Québec, 1919-1934), 2: 185 sqq. Voir aussi la note 160 . 
noms des principales familles canadiennes. ${ }^{160}$ On compte même parfois jusqu'à trois membres d'une même famille (v.g. les de Léry), dont l'un ou l'autre peut cumuler plusieurs emplois. Or cette impression se trouve vérifiée par notre exercice qui établit une pondération généralement dominante des nobles, spécialement dans les catégories de postes et de pensions de plus de $£ 100$ st. Il y a cependant un accroissement très net de la participation des non-nobles. Vers la fin de la période, les deux groupes s'équivalent à peu près en ce qui concerne le nombre de places. C'est au niveau des postes les plus rémunérateurs que transparaît clairement la force réelle de l'aristocratie locale: les rapports en sa faveur sont de l'ordre de 3 ou 4 contre 1 .

Ainsi, les non-nobles grignotent une portion grandissante de la liste civile, mais seulement aux bas échelons et à cause de la prolifération de petits emplois (v.g. les maîtres d'école). La noblesse, elle, préserve son ascendant en monopolisant la presque totalité des postes et des pensions d'importance et en se maintenant assez bien dans la dernière catégorie d'emplois (moins de 1100 st.) grâce à l'expansion de la milice à compter de 1804$1805 . .^{161}$

Enfin, l'on peut extraire du tableau VIII une conclusion additionnelle. En effet, comment ne pas constater la remarquable stabilité des emplois occupés par les professionnels canadiens-français (nobles et non-nobles) tant dans la liste civile en général que dans la fonction publique proprement dite et surtout dans les hauts postes. De ce côté, on perçoit un pla-

${ }^{160} \mathrm{La}$ liste civile est un véritable Who's Who de l'ancienne aristocratie. S'y casent les de St-Ours, de Longueuil, Baby, de Bonne, de Lanaudière (Charles et Xavier), Taschereau, de Rocheblave, Cugnet, de Boucherville (R.-A. et P.), Duchesnay, Montizambert, de la Chevrotière, de Chambault, de Léry (J.-G.-C., L.-R.-C. et C.-E.), du Vergé, La Corne St. Luc, de Louvière, de Salaberry, d'Estimauville, de Goutin, de st. Vilmé, de Varenne, de Chambault, Voyer, de Monviel, etc.). Même les observateurs contemporains non engagés le remarquent. Ainsi, Selkirk écrit dans son journal: "... both Ld. Dorchester and Sr. Robt. Milnes have been partial to the Canadian gentry". (Cité dans P.C.T. White, éd., Lord Selkirk's Diary 1803-1804 (Toronto, 1958), 219). Dès que meurt un membre de ce cercle restreint, ses rejetons et ceux des autres bonnes familles se disputent l'emploi vacant. Par exemple, à la suite de la mort coup sur coup de deux titulaires du poste de grand voyer du district de Québec, en 1809 et 1810 , bon nombre de Canadiens postulent l'emploi (Vassal de Monviel, O. Perrault, P. Lacroix, L. J. Perrault, etc.), mais c'est finalement J.-B. d'Estimauville qui hérite de cette place (APC, R.G.4, Al, S, 80: 67 sqq.).

161 Il y a un adjudant de milice canadien-français en 1804 , treize, en 1812 , presque tous des "nobles" (V. de Monviel, X. de Lanaudière, A. J. Duchesnay, L. Montizambert, J.-B. d'Estimauville, L.-R.-C. de Léry, etc.). 


\section{TABLEAU VIII}

Origine sociale des Canadiens français dans la liste civile *

$$
\text { (1794-1812) }
$$

(1)

\begin{tabular}{lcccrcrr} 
& \multicolumn{6}{c}{ Liste civile globale } \\
& $\overline{\mathrm{P}}$ & $\mathrm{P}$ & $\mathrm{T}$ & $\overline{\mathrm{P}}$ & $\mathrm{P}$ & \multicolumn{1}{c}{$\mathrm{T}$} \\
1794 & 30 & 4 & 34 & 4 & 1 & 5 \\
1797 & 38 & 2 & 40 & 13 & 4 & 17 \\
1800 & 37 & 2 & 39 & 17 & 4 & 21 \\
1803 & 40 & 3 & 43 & 26 & 7 & 33 \\
1806 & 42 & 3 & 45 & 33 & 6 & 39 \\
1809 & 42 & 2 & 44 & 40 & 4 & 44 \\
1812 & 33 & 2 & 35 & 36 & 5 & 41
\end{tabular}

\section{(2)}

Fonction publique réelle ** $\overline{\mathrm{P}} \quad-\mathrm{NN}$

\section{(3)}

Postes et pensions de plus de $£ 100$ st.

$$
\begin{array}{llllllllllll}
\overline{\mathrm{P}} & \mathrm{P} & \mathrm{T} & \overline{\mathrm{P}} & \mathrm{P} & \mathrm{T} & \overline{\mathrm{P}} & \mathrm{P} & \mathrm{T} & \overline{\mathrm{P}} & \mathrm{P} & \mathrm{T}
\end{array}
$$$$
\begin{array}{llllllllllll}
13 & 4 & 17 & 3 & 1 & 4 & 13 & 2 & 15 & 0 & 1 & 1
\end{array}
$$$$
\begin{array}{lll}
16 & 2 & 18
\end{array}
$$$$
5 \quad 4 \quad 9
$$$$
\begin{array}{lll}
14 & 2 & 16
\end{array}
$$$$
\begin{array}{lll}
8 & 4 & 12
\end{array}
$$$$
\begin{array}{lll}
13 & 2 & 15
\end{array}
$$

$\begin{array}{lll}13 & 3 & 16\end{array}$

$\begin{array}{lll}23 & 3 & 26\end{array}$

$\begin{array}{lll}14 & 7 & 21\end{array}$

$\begin{array}{lll}14 & 2 & 16\end{array}$

$\begin{array}{lll}14 & 2 & 16\end{array}$

$\begin{array}{lll}16 & 6 & 22\end{array}$

$\begin{array}{lll}17 & 2 & 19\end{array}$

$\begin{array}{llllll}22 & 2 & 24 & 29 & 4 & 33\end{array}$

$\begin{array}{lll}28 & 2 & 30\end{array}$

$\begin{array}{lll}34 & 5 & 39\end{array}$

$\begin{array}{lll}16 & 2 & 18\end{array}$

14216

$\begin{array}{lll}0 & 3 & 3\end{array}$

\begin{tabular}{|c|c|}
\hline \multicolumn{2}{|c|}{$(4)$} \\
\hline \multicolumn{2}{|c|}{ Pensions } \\
\hline $\mathrm{N}$ & NN \\
\hline 17 & 1 \\
\hline 22 & 5 \\
\hline 22 & 8 \\
\hline 21 & 11 \\
\hline 19 & 12 \\
\hline 19 & 11 \\
\hline 5 & 2 \\
\hline
\end{tabular}

$\begin{array}{lll}2 & 3 & 5\end{array}$

Source: JCA $B C$, 1794-1813.

${ }^{*} \mathrm{~N}=$ nobles. $\mathrm{NN}=$ non-nobles. $\mathrm{P}=$ professionnels. $\overline{\mathrm{P}}=$ non-professionnels.

** C'est-à-dire les emplois civils sans les pensions ni les sommes attribuées à des missionnaires. Cependant, ils comprennent les maîtres d'école. Si les additions des colonnes (2) et (4) n'arrivent pas toujours aux totaux de la colonne 1, c'est justement à cause de l'exclusion des missionnaires. 
fonnement des ouvertures pour les professionnels dès le tournant du XIX ${ }^{e}$ siècle. ${ }^{162}$

Ce croquis trop rapide des composantes sociales du groupe canadien-français dans la liste civile, permet de mieux cerner les fondements démographiques et économiques des tensions sociales (et même politiques) à l'intérieur du groupe canadienfrançais. Il confirme que dans le Bas-Canada, la part du lion dans les places et les pensions octroyées aux Canadiens français, est réservée à l'aristocratie locale. Cependant, le caractère restreint des données nous interdit de nous aventurer plus loin pour le moment. Nous retiendrons toutefois ces quelques jalons lors de notre interprétation ultérieure (section VII) de nos analyses plus poussées de certaines autres dimensions de la liste civile.

Il nous reste le matériau principal à travailler: l'analyse des salaires et des pensions dans leurs composantes ethniques et régionales.

\section{(3) Les pensions}

Il n'a pas été possible d'anatomiser les pensions comme nous l'aurions voulu, à cause de l'imprécision de la liste civile relativement à la localisation exacte des pensionnés. Nous avons dû classer les trois quarts des pensions dans la catégorie "région inconnue". L'autre quart nous a paru un échantillon trop restreint pour en déduire des observations valables. Par contre, les données à propos de l'identité ethnique des pensionnés sont assez précises pour autoriser une analyse fouillée.

Ainsi que le montre le tableau IX, les Canadiens anglais ne moissonnent que le cinquième des pensions (en nombre) au début de la période, encore qu'il s'agisse environ du quart en valeur. En 1794, la moyenne des pensions versées aux Canadiens français surplombe de $40 \%$ celle des pensions payées aux Canadiens anglais. Mais dès les années suivantes, cette moyenne s'effondre à environ $75 \%$ de celle des pensions des Canadiens anglais, et l'écart continue de s'élargir avec le temps: en 18081809, la moyenne des pensions des Canadiens français n'est plus que de $15 \%$ approximativement de celle des pensions allant à des Canadiens anglais!

$162 \mathrm{~F}$. Ouellet a soulevé le problème d'une absence générale de débouchés pour les professionnels (Louis-Joseph Papineau, un être divisé (Ottawa, 1960), 8-9; Histoire économique et sociale du Québec, 1760-1850 (Montréal, 1966), chap. V, VI et VII, passim). Nous discuterons davantage ce problème dans la section VII. 
TABLEAU IX

Nombre, somme et moyenne des pensions par groupe ethnique *

(1794-1812)

Nombre de pensions Somme des pensions

CF CA

\begin{tabular}{rrr}
1794 & 38 & 11 \\
5 & 36 & 11 \\
6 & 36 & 10 \\
7 & 38 & 8 \\
8 & 37 & 8 \\
9 & 37 & 8 \\
1800 & 37 & 9 \\
1 & 39 & 9 \\
2 & 39 & 10 \\
3 & 42 & 9 \\
4 & 41 & 9 \\
5 & 38 & 11 \\
6 & 37 & 13 \\
7 & 38 & 13 \\
8 & 36 & 17 \\
9 & 38 & 17 \\
1810 & 8 & 14 \\
1 & 8 & 14 \\
$\mathbf{2}$ & 7 & 14 \\
\hline
\end{tabular}

$\mathrm{CF}$

$1201.0 * *$

1028.6

1027.8

1107.2

880.3

985.2

980.1

951.5

965.5

1037.8

900.4

835.4

823.9

742.0

704.0

817.2

381.8

411.5

471.6
CA

245.6

400.3

391.1

441.6

444.3

445.6

539.0

557.7

961.5

1353.5

1351.1

1770.3

1736.2

1935.0

2063.7

2485.6

2630.3

2765.0

1990.0
Moyenne des pensions

CF

CA

31.6

28.6

22.3

$28.5 \quad 39.1$

$29.1 \quad 55.2$

$23.8 \quad 55.5$

$26.6 \quad 55.7$

$26.5 \quad 59.9$

$24.4 \quad 62.0$

$24.7 \quad 96.1$

$24.7 \quad 150.4$

$22.0 \quad 150.1$

$22.0 \quad 160.9$

$22.3 \quad 133.5$

$19.5 \quad 148.8$

$19.5 \quad 121.4$

$21.5 \quad 146.2$

$47.7 \quad 187.9$

$51.4 \quad 197.5$

$67.4 \quad 142.1$

Source: JCABC, 1794-1813.

* L'unité monétaire utilisée est la livre sterling exprimée en notation décimale.

** Dans ce tableau comme dans les autres, les décimales ont été arrondies de façon à les réduire à une seule.

Malgré la remarquable stabilité du nombre absolu des pensions des Canadiens français, entre 1794 et 1809, on enregistre une chute de plus de $30 \%$ dans la somme allouée à ces pensions avec, comme conséquence, un affaissement du même ordre dans la moyenne des pensions. En fait, il se produit un renversement complet de la situation en ce qui concerne les sommes départies aux deux groupes ethniques. En 1794-95, les Canadiens français emportent les 5/6 de la somme totale; en 1809, ce sont les Canadiens anglais qui en engloutissent les $3 / 4$.

En nombre, les Canadiens français pensionnés demeurent la majorité jusqu'en 1809 (les $4 / 5$ environ en 1797 , les $3 / 4$ au 
milieu de la période et encore au moins les $2 / 3$ en 1809). Cependant, ils sont déplacés vers des pensions de niveau moins élevé, alors que les Canadiens anglais augmentent peu en nombre mais ravissent rapidement la plus grande partie des sommes affectées aux pensions (le tiers en 1797, la moitié en 1803, les deux tiers en 1806 et les trois quarts en 1809). La coupure brutale de 1810, au plus fort de la crise sous Craig, accentue s'il se peut le renversement: les petites pensions tombent et le groupe canadien-anglais domine alors la scène des pensions tant en nombre qu'en valeur $-2 / 3$ des pensions, $7 / 8$ des montants.

Les pensionnés canadiens-français sont pour la plupart des membres de la noblesse seigneuriale et militaire ${ }^{163}$ que le gouvernement indemnise pour des services rendus soit en 1764 lors de la révolte de Pontiac, soit lors de la guerre contre les EtatsUnis. ${ }^{164}$ Il y a aussi des pensions charitables à des "gentilshommes" et à des "dames canadiennes" "réduits à la détresse" et à "l'indigence": ${ }^{165}$ généralement de petites sommes, £20 ou moins. Mais à mesure qu'on se rapproche de 1812 , les pensions en viennent à récompenser des services plus "civils". ${ }^{166} \mathrm{La}$ proportion des pensions aux nobles, bien que toujours majoritaire, décroît légèrement dès $1797 .{ }^{167}$

Chez les Canadiens anglais, les pensionnés sont surtout des hauts fonctionnaires dévoués et leurs survivants. Plus nombreux dans le fonctionnarisme, ils doivent forcément faire irruption, tôt ou tard, au niveau des pensions. Au cours de la période, ils en viennent à remplacer les Canadiens français décédés; et vu

163 Voir tableau VIII.

164 Entre 1774 et 1783 , particulièrement durant l'invasion américaine (1775-1776) et au siège de Québec.

165 Cette indigence était parfois fort pénible, surtout pour les personnes de bonnes familles. L'abbé Robin parle de "Mme Launière et ses trois Demoiselles, .... dans un état de détresse qui fait de la peine..." (abbé F. Robin à Louis de Salaberry, Québec, 23 janvier 1799, APQ, gr. coll., papiers de Salaberry, 1: s.p.).

166 Services dans le fonctionnarisme ou services politiques, culturels, etc. V.g. le professeur L.-G. Labadie, chantre des vertus de l'Angleterre et du gouvernement, reçoit une pension de $£ 20$ pour "services rendus au gouvernement" en 1801. D'autres pensions sont versées directement à partir de la caisse militaire, v.g. à Louis Vincent, pour son dévouement comme maître d'école auprès des enfants hurons, à Lorette, $£ 10$ c. par année (APC, R.G.8, C, 0: 68.). De plus, certains administrateurs comme Dorchester et Milnes croient en la nécessité d'une aristocratie locale forte et bien nantie pour contrebalancer l'élément "populaire" qui domine l'Assemblée (v.g. DC II, 228-245). 167 Voir tableau VIII. 
l'importance des fonctions qu'ils ont occupées, ils retirent des pensions parfois fort élevées. ${ }^{168}$

Ce qui étonne surtout, c'est la rapidité et le caractère radical du renversement. Pour les pensions, le mécanisme de redistribution joue nettement et de plus en plus en faveur des Canadiens anglais. D'autre part, l'inversion frappe plus durement et directement l'ancienne élite seigneuriale et militaire, qui a profité surtout des pensions au début de la période. Les non-nobles canadiens-francais n'y ont jamais compté pour beaucoup. Ce tarissement relatif se conjugue au grossissement des familles nobles pour empirer leur situation.

Nous ne tenterons pas ici de déchiffrer la nature du mécanisme qui sous-tend ce renversement des agrégats: cette question nécessite une analyse plus fine des données et nous la reportons à la section VI.

\section{(4) Les salaires}

Du côté de la fonction publique, une analyse plus précise est possible grâce à la meilleure qualité des renseignements. D'une façon globale, on assiste à un accroissement continu et graduel du nombre des emplois à mesure que la population de la colonie et ses besoins collectifs augmentent. Entre 1790 et 1812, la population du Bas-Canada double; ${ }^{169}$ le nombre des emplois publics et les sommes qu'ils représentent font presque le même bond. ${ }^{170}$

${ }^{168}$ V.g. l'ancien juge en chef Osgoode qui, après 1802, reçoit une pension de $£ 800$ st., puis de $£ 400$ st.; Thomas Dunn, juge et président du Conseil exécutif, une pension de $£ 500$ st.

169 Voir note 73.

170 Toutefois, le nombre des emplois doit être pondéré par celui des cumuls (voir tableaux VI et VII) pour en tirer une idée plus juste des effectifs réels de la fonction publique. L'accroissement en valeur de la liste civile tient à la fois à la multiplication des emplois et à la hausse des salaires, surtout sensible dans les catégories supérieures. Bien que cela n'entre pas dans le cadre de l'étude quantitative actuelle, il serait intéressant de comparer les augmentations de salaires selon les catégories, au long de la période. A un premier coup d'œil, l'écart semble s'accroître entre petits, moyens et hauts salariés. Pour une première ébauche d'une semblable étude, voir G. Paquet et J.-P. Wallot, "Lower Canada, 1792-1812 [...]", tableau X. D'autre part, l'expansion des emplois s'accélère en 1805 et en 1811. Alors, plusieurs nouveaux postes se créent: assistants-secrétaires at commis, maîtres de hâvre à Québec et à Montréal (dans le premier cas, au salaire de $£ 100$ st. par année en 1806 , $£ 160$ st. en 1811), clerc du marché de Québec, etc. Les progrès sur tous les plans créent de nouveaux besoins ou valorisent d'anciennes fonctions longtemps stagnantes sur le plan salarial. 
TABLEAU $\mathrm{X}$

Nombre de salaires ventilés par catégories et ethnies

(1794-1812)

\begin{tabular}{|c|c|c|c|c|c|c|c|c|c|c|c|c|c|c|c|}
\hline & \multicolumn{2}{|c|}{$<100$} & \multicolumn{2}{|c|}{$100-200$} & \multicolumn{2}{|c|}{$200-300$} & \multicolumn{2}{|c|}{$300-500$} & \multicolumn{2}{|c|}{$500-1000$} & \multicolumn{2}{|c|}{$1000+$} & \multicolumn{2}{|c|}{ Total } & \multirow{2}{*}{$\begin{array}{c}\text { Total } \\
\text { (incluant } \\
\text { les in- } \\
\text { connus) }\end{array}$} \\
\hline & $\mathrm{CF}$ & $\mathrm{CA}$ & $\mathrm{CF}$ & $\mathrm{CA}$ & $\mathrm{CF}$ & $\mathrm{CA}$ & $\mathrm{CF}$ & $\mathrm{CA}$ & $\mathrm{CF}$ & $\mathrm{CA}$ & $\mathrm{CF}$ & $\mathrm{CA}$ & $\mathrm{CF}$ & $\mathrm{CA}$ & \\
\hline 1794 & 18 & 39 & 7 & 19 & 2 & 7 & 2 & 7 & 1 & 4 & 0 & 2 & 30 & 78 & 110 \\
\hline 1795 & 16 & 39 & 8 & 20 & 4 & 6 & $\overline{0}$ & 4 & 2 & 6 & 0 & $\overline{2}$ & 30 & 77 & 109 \\
\hline 1796 & 16 & 39 & 9 & 22 & 1 & 5 & 1 & 6 & 3 & 4 & 0 & 3 & 30 & 79 & 111 \\
\hline 797 & 18 & 41 & 9 & 20 & 1 & 6 & 1 & 6 & 3 & 7 & 0 & 2 & 32 & 82 & 117 \\
\hline 798 & 16 & 40 & 9 & 19 & 2 & 6 & 1 & 6 & 3 & 5 & 0 & 3 & 31 & 79 & 113 \\
\hline 1799 & 18 & 40 & 8 & 19 & $\overline{2}$ & 7 & 1 & 8 & 3 & 6 & 0 & 3 & 32 & 83 & 118 \\
\hline 800 & 20 & 41 & 10 & 23 & 2 & 7 & 1 & 8 & 3 & 4 & 0 & 4 & 36 & 87 & 126 \\
\hline 801 & 16 & 43 & 11 & 21 & 2 & 6 & 0 & 8 & 4 & 5 & 0 & 4 & 33 & 87 & \\
\hline & 16 & 49 & 13 & 16 & 2 & 7 & 0 & 8 & 4 & 6 & 0 & 2 & 35 & 88 & 127 \\
\hline 30 & 20 & 49 & 12 & 21 & 1 & 4 & 2 & 7 & 3 & 5 & 0 & 4 & 38 & 90 & 128 \\
\hline 1804 & 29 & 42 & 13 & 19 & 1 & 6 & $\overline{1}$ & 8 & 4 & 4 & 0 & 4 & 4 & 83 & 134 \\
\hline 100 & 30 & 58 & 15 & 19 & 2 & 4 & 1 & 8 & 4 & 6 & 0 & 4 & 52 & 99 & 16 \\
\hline 806 & 35 & 54 & 14 & 20 & 2 & 8 & 1 & 7 & 4 & 5 & 0 & 5 & 56 & 99 & 163 \\
\hline 1807 & 38 & 60 & 13 & 20 & 2 & 8 & 1 & 8 & 4 & 7 & 0 & 7 & 5 & 110 & 174 \\
\hline & 49 & 65 & 12 & 28 & $\overline{2}$ & 7 & 4 & 8 & 4 & 4 & 0 & 3 & 71 & 115 & 190 \\
\hline & 42 & 60 & 9 & 27 & 4 & 6 & 2 & 8 & 4 & 4 & 0 & 5 & 61 & $\begin{array}{l}110 \\
110\end{array}$ & $\begin{array}{l}176 \\
\end{array}$ \\
\hline & 47 & 59 & 12 & 24 & 5 & 5 & 2 & 12 & 4 & 5 & 0 & 4 & 7 & 109 & 185 \\
\hline 10. & 53 & 66 & 10 & 26 & 6 & 6 & 3 & 13 & 3 & 5 & 0 & 4 & 75 & 120 & 201 \\
\hline 1812 & 57 & 79 & 11 & 22 & 5 & 9 & 3 & 10 & 3 & 7 & 0 & 3 & 79 & 130 & 215 \\
\hline
\end{tabular}

Source: JCA BC, 1794-1813. 
TABLEAU XI

Nombre, somme et moyenne des salaires selon les groupes ethniques (1794-1812)

\begin{tabular}{|c|c|c|c|c|c|c|c|c|c|}
\hline \multicolumn{4}{|c|}{ Nombre de salaires } & \multicolumn{3}{|c|}{ Somme des salaires } & \multicolumn{3}{|c|}{ Moyenne des salaires } \\
\hline & c. ${ }^{+}$ & CF & $\mathrm{CA}$ & INC. & $\mathrm{CF}$ & $\mathrm{CA}$ & INC. & $\mathrm{CF}$ & $\mathrm{CA}$ \\
\hline 94 & 2 & 30 & 78 & 84.6 & 3204.3 & 13165.4 & 42.3 & 106.8 & 168. \\
\hline & 2 & 30 & 77 & 84 & 347 & 134 & 42.3 & & \\
\hline & 3 & 30 & 79 & 116.6 & 3835.4 & 14282.3 & 38.9 & 127.8 & \\
\hline$\gamma$ & 3 & 32 & 82 & 188 & 368 & 144 & 63.0 & 115 & \\
\hline 8 & 3 & 31 & 79 & 168.3 & 4009.3 & & 56.1 & 129.3 & \\
\hline & 3 & 32 & 83 & 142.3 & 39 & & 47.4 & 12 & \\
\hline 800 & 3 & 36 & 87 & 0 & & & 55.0 & 12 & \\
\hline 1 & 3 & 33 & 87 & 229.8 & 50 & & 76.6 & 15 & \\
\hline & 4 & 35 & 88 & 310.3 & 529 & & 77.6 & 15 & 215 \\
\hline 3 & 0 & 38 & 90 & - & 533 & 203 & & 14 & 226 \\
\hline 4 & 3 & 48 & 83 & 78.0 & & & 26.0 & 12 & \\
\hline 5 & 9 & 52 & 99 & 159.0 & 614 & & 17.7 & 118.2 & 18 \\
\hline & 8 & 56 & 99 & 193.9 & & & & & \\
\hline & 6 & 58 & 110 & 148.7 & 63 & & 24.8 & 108.7 & 25 \\
\hline & 4 & 71 & 11 & 21.0 & & & 30.2 & & \\
\hline & 5 & 61 & 11 & 127.1 & & & 25.4 & & \\
\hline 10 & 6 & 70 & 10 & 153.5 & & & 25.6 & & \\
\hline & 6 & 75 & 12 & 143.3 & 79 & 22 & 23.9 & 106.3 & \\
\hline 2 & 6 & 79 & 13 & 9.3 & 7552.2 & 24 & 28.2 & 95.6 & \\
\hline
\end{tabular}

Source: JCABC, 1794-1813.

+ INC. = inconnus.

Jamais, au cours de cette période, les Canadiens français qui pourtant constituent environ $90 \%$ de la population bascanadienne, ne réussissent-ils à occuper même $40 \%$ des emplois. Ils en détiennent un peu plus du quart, de 1794 à 1801; puis s'amorce une remontée qui débouche, entre 1804 et 1812, sur un pourcentage oscillant entre 34 et $40 \%$ (tableau X). En valeur, parmi les salaires identifiés en fonction de l'ethnie, la portion propre aux Canadiens français se situe à un niveau encore plus bas: entre 20 et $25 \%$ du total (tableau XI).

Aux fins d'analyse, nous avons réparti les salaires en 6 catégories. Au-delà de la simple arithmétique, cette ventilation décompose le bloc des salaires en types d'emploi qui ont des valeurs sociales, politiques et administratives fort diverses.

Les postes de $£ 1,000$ st. ou plus sont très rares. Il s'agit de ceux de gouverneur, lieutenant-gouverneur, juge en chef de 
Québec et juge en chef de Montréal (à compter de 1803). La seconde catégorie, de $£ 500$ à $£ 999$ st., comporte les emplois judiciaires dans la province (cours du banc du roi) et une sinécure honorifique (grand voyer de la province, détenu par un noble canadien-français durant toute la période étudiée). La troisième catégorie, de $£ 300$ à $£ 499$ st., s'avère la plus importante au point de vue de la marche de l'administration coloniale: elle inclut les principaux postes de commande (receveur général, inspecteur des comptes provinciaux, procureur général, arpenteur général, greffier du Conseil exécutif, secrétaire de la province, commissaire des sauvages - poste qui passe très tôt dans les dépenses militaires -, parfois le percepteur des douanes à Québec, payé à pourcentage des droits perçus, etc.). Ce sont ces postes qui font fonctionner la colonie au jour le jour. En outre, plusieurs de leurs titulaires cumulent d'autres fonctions lucratives ou encore touchent des honoraires additionnels pour "services divers", des allocations de dépenses, etc. La quatrième catégorie, de $£ 200$ à $£ 299$ st., comprend des postes moins importants, surtout de greffiers, secrétaires et parfois d'assistants de ceux de la catégorie 3 (solliciteur général, juge de Gaspé, secrétaire du gouverneur, inspecteur général des forêts, greffiers du Conseil législatif et de l'Assemblée, traducteur français du gouverneur et du Conseil exécutif, juge de la cour de viceamirauté, assistant-secrétaire de la province, etc.). La cinquième catégorie, de $£ 100$ à $£ 199$ st., rassemble les emplois subalternes (v.g. commis de bureau, assistants-secrétaires, assistants-arpenteurs, traducteurs, greffiers, interprètes, shérifs). La dernière catégorie - celle des emplois de moins de $£ 100$ - groupe les tâches de bureau de niveau inférieur (commis, clercs, inspecteurs de douanes, etc.), les emplois manuels et menues occupations (v.g. crieurs de cours, portiers, gardiens de prison, hommes et femmes de ménage, ramoneurs de cheminées - affublés du titre ronflant d" "inspecteurs de feu" -, interprètes de cours), et un nombre croissant de postes plus relevés, mais peu rémunérateurs et parfois à temps partiel (v.g. missionnaires, instituteurs - un en 1802, plus d'une douzaine en 1812 - , adjudants de bataillons de milice - un en 1804, 18 en 1812).

Un examen rapide du tableau $\mathrm{X}$ révèle que les Canadiens français sont en minorité dans toutes les catégories, bien que davantage dans certaines que dans d'autres. Aucun d'entre eux ne gagne $£ 1,000$ et plus. Dans la seconde catégorie, ils s'affichent relativement forts et parfois égalent le nombre des Britanniques (v.g. en 1804, 1808 et 1809) : il s'agit en effet de fonctions judiciaires, hormis pour la sinécure de grand voyer, tenue par 
Charles de Lanaudière jusqu'en 1811, puis par François Baby, trop vieux pour demeurer adjudant général de milice et remplacé à ce dernier poste par un autre noble, Vassal de Monviel. Il faut absolument quelques juges canadiens-français, généralement deux ou trois, afin d'interpréter les lois civiles françaises et d'éclairer les juges britanniques, pour la plupart peu versés dans les lois canadiennes. ${ }^{171}$ Il s'agit ici véritablement d'une "fonction" au sens le plus rigoureux du terme.

C'est dans les postes clé de la catégorie des $£ 300-500$, ceux qui président à l'administration concrète de la colonie et exécutent la politique gouvernementale, que se fait le plus sentir l'absence quasi totale de Canadiens francais (1 ou 2 d'entre eux contre 7 ou 8 Britanniques). Malgré les apparences (v.g. en 1808,1811 et 1812 ), ils n'y comptent au plus que deux membres importants: Joseph Bouchette, promu arpenteur général en 1804, et François Baby, dont le salaire à titre d'adjudant général de la milice est relevé, en 1808, jusqu'à cette catégorie - il passe de $£ 91$ à $£ 320$ st. ! - , bien que cette fonction devienne active surtout à compter de 1811. Dans les quelques autres cas, il s'agit soit du juge provincial des Trois-Rivières, avant la hausse de son traitement jusqu'à $£ 500$ en 1801 , soit d'une partie du salaire d'un juge de la cour du banc du roi qui n'a pas siégé une année complète.

Dans la catégorie des $£ 200-300$, également très importante pour la bonne marche de la colonie, car on y trouve les assistants immédiats des chefs de service, les Canadiens français sont encore carrément en minorité sauf pour les années 1810 et 1811. Leur nombre varie de façon assez brusque (il y a parfois des parties de salaires supérieurs, versés pour moins de douze mois), partant du quart, montant au tiers, puis dégringolant aussi bas qu'au cinquième pour rebondir ensuite à moins de la moitié, hormis en 1810 et en 1811. Encore ici, si l'on s'arrête aux années record (1810-1811), on se rend compte jusqu'à quel point les emplois des Canadiens français tiennent à leur présence majoritaire dans la province et à la nécessité pour l'Etat de transiger avec eux: $X$. de Lanaudière, traducteur et secrétaire français de la province, C.-E. de Léry, assistant-greffier et traducteur du Conseil législatif, P.-E. Desbarats, assistant-greffier et traducteur de la Chambre d'Assemblée. Un autre poste, celui de J. Planté, agrège en fait des commissions dues pour plusieurs années et réglées d'un seul coup. Seuls deux Canadiens français

${ }_{171}$ Sur le fouillis dans l'administration de la justice, voir J.-P. Wallot, "Plaintes contre l'administration de la justice (1807)", $R H A F, 19$ (19651966) : 551-560; 20 (1966-1967) : 28-43, 281-290, 366-379. 
s'adonnent à des occupations différentes et d'ailleurs créées en 1810 par Craig: J. Mondelet, juge de police à Montréal, et Louis de Salaberry, inspecteur des forêts, apparemment une sinécure. La même catégorie, chez les Britanniques, comprend les fonctions de solliciteur général, de juge de police à Montréal, d'auditeur des patentes sur les terres, de secrétaire du gouverneur (poste extrêmement important et qui permet en outre, comme dans le cas de Ryland, d'en cumuler deux ou trois autres), et du juge de la cour de vice-amirauté. Si l'on fusionne les catégories 3 et 4 , même si celle-ci tempère la disproportion trop forte de celle-là, on ne peut s'empêcher de conclure à l'absence notable de Canadiens français dans les postes de commande.

Plus étonnante nous apparaît la même infériorité (en nombre et en valeur des sommes impliquées) des Canadiens français dans la catégorie des $£ 100-200$, celle des employés subalternes, surtout dans divers bureaux. Ici encore, l'on perçoit la même "vocation" des groupes ethniques: les Canadiens français remplissent surtout des fonctions de traducteurs, greffiers, interprètes, etc., hormis pour trois ou quatre conseillers exécutifs. Ils font le pont entre les services de l'Etat et la population. Par contre, la plupart des commis, assistants-secrétaires, shérifs, assistants-arpenteurs se recrutent chez les Britanniques. Ainsi, en 1810, le bureau du secrétariat de la province et du gouverneur ne compte que des Britanniques (4), sauf pour le traducteur des proclamations. Par contre, la même année, quelques Canadiens français assument des fonctions plus intégrées à la vie économique et politique: le maître de havre à Québec - la plupart des pilotes sur le Saint-Laurent sont d'ailleurs Canadiens -, les grands voyers des districts de Québec et de Montréal, toujours des Canadiens français de la noblesse, le surintendant des maisons de poste provinciales, également un noble.

Mais la plus grande surprise survient dans la dernière catégorie. Sans doute, les Canadiens français devraient-ils abonder au moins dans ces situations inférieures de commis, messagers, portiers, crieurs, femmes de ménage, etc. Pas davantage. Ici encore se manifeste leur absence, bien qu'il y ait amélioration relative de leur position au cours de ces années. Certes, c'est sans doute ici qu'ils sont mieux partagés. Mais leur proportion, dans les meilleures années, ne dépasse guère $40 \%$ et sombre parfois en dessous du tiers pendant une étonnante période creuse, entre 1795 et 1805 . Par conséquent, même le menu peuple canadien-français ne bénéficie pas d'un nombre d'emplois proportionnel à son importance numérique dans la province. Bien plus, même si elle ne constitue qu'une faible minorité dans 
l'ensemble de la population, l'élite canadienne-française, malmenée aux autres niveaux, empiète jusque dans ce domaine qu'on eût cru devoir appartenir au peuple. Même la multiplication des emplois, à compter de 1809 , apporte peu aux classes populaires: ce gonflement est dû à l'excroissance du corps de milice, dont les officiers sont choisis par le gouverneur presque exclusivement dans la noblesse.

Une comparaison des masses salariales et des moyennes de salaires entre groupes ethniques confirme cet état d'infériorité économique des Canadiens français dans la fonction publique. Comme l'indique le tableau XI, moins du quart de la masse salariale leur revient. En ce qui concerne la moyenne des salaires, la situation relative des Canadiens français semble même se détériorer avec le temps. Alors qu'au cours de la première moitié de la période, la moyenne des salaires payés pour des fonctions détenues par des Canadiens français se maintient autour des $2 / 3$ de la moyenne correspondante pour les Canadiens anglais, le pourcentage s'incline à environ $55 \%$ au cours de la seconde moitié. L'écart croissant est fort clair (voir section VI, graphique III).

Cet écart grandissant nous conduit à sonder les autres principes de classification qui pourraient nous permettre de saisir les mouvements de restructuration à l'intérieur de la fonction publique. Nous avons décanté certains ajustements dans la configuration géographique des emplois et des salaires. Ce qui étonne d'abord, c'est la stabilité relative de la structure des emplois dans l'ensemble des diverses régions. Tant à Québec, à Montréal qu'ailleurs, le nombre des emplois double ou à peu près entre 1795 et 1812. Cependant, les niveaux moyens de salaire varient d'une façon prononcée et assez erratique d'une région à l'autre. La moyenne des salaires à Québec est plus élevée qu'à Montréal, sauf pour 1806 (tableau XII). Cependant la différence est très variable. Nous avons fondu toutes les autres régions connues ensemble, à cause du petit nombre d'observations pour chacune.

Pour départager de façon préliminaire les tendances sousjacentes à cette double classification par régions et par ethnies, nous avons construit un tableau qui en isole les 4 composantes (Canadiens français-Canadiens anglais, Québec-Montréal). Les résultats sont intéressants. On constate la différence énorme et soutenue entre les moyennes de salaire des groupes ethniques à Québec (voir section VI, graphique IV), par opposition au renversement complet des positions des Canadiens français et 
TABLEAU XII

Nombre, somme et moyenne des salaires par région (1794-1812)

\begin{tabular}{|c|c|c|c|c|c|c|c|c|c|}
\hline & \multicolumn{3}{|c|}{ Québec } & \multicolumn{3}{|c|}{ Montréal } & \multicolumn{3}{|c|}{ Autres régions connues } \\
\hline & $\begin{array}{c}\text { Nom- } \\
\text { bre }\end{array}$ & Total & Moyenne & $\begin{array}{c}\text { Nom- } \\
\text { bre }\end{array}$ & Total & Moyenne & $\begin{array}{c}\text { Nom- } \\
\text { bre }\end{array}$ & Total & Moyenne \\
\hline 1794 & 63 & 11166.7 & 177.2 & 24 & 1538.3 & 64.1 & 18 & 3225.0 & 179.2 \\
\hline 5 & 59 & 10103.7 & 171.2 & 30 & 3131.6 & 104.4 & 14 & 1212.7 & 86.6 \\
\hline 6 & 66 & 12505.9 & 189.5 & 28 & 4155.4 & 148.4 & 17 & 1437.9 & 84.6 \\
\hline 7 & 69 & 12263.6 & 177.7 & 31 & 4679.3 & 150.9 & 16 & 1333.4 & 83.3 \\
\hline 8 & 64 & 12950.5 & 202.4 & 30 & 4571.5 & 152.4 & 17 & 1408.8 & 82.9 \\
\hline 9 & 71 & 14069.8 & 198.2 & 29 & 4593.5 & 158.4 & 18 & 1389.8 & 77.2 \\
\hline 300 & 68 & 16825.6 & 247.4 & 36 & 4824.3 & 134.0 & 22 & 1519.4 & 69.1 \\
\hline 1 & 72 & 17348.5 & 241.0 & 31 & 5474.9 & 176.6 & 20 & 1732.4 & 86.6 \\
\hline 2 & 78 & 18297.3 & 234.6 & 31 & 4603.4 & 148.5 & 17 & 1624.6 & 95.6 \\
\hline 3 & 76 & 17302.8 & 227.7 & 30 & 5920.8 & 197.4 & 22 & 2486.0 & 113.0 \\
\hline 4 & 80 & 17776.9 & 222.2 & 33 & 5853.5 & 177.4 & 21 & 2144.1 & 102.1 \\
\hline 5 & 99 & 17436.5 & 176.1 & 36 & 6097.2 & 169.4 & 25 & 1571.1 & 62.8 \\
\hline 6 & 96 & 17352.1 & 180.8 & 41 & 7655.4 & 186.7 & 25 & 2145.5 & 85.8 \\
\hline 7 & 102 & 27181.5 & 266.5 & 45 & 5278.8 & 117.3 & 26 & 1673.0 & 64.3 \\
\hline 8 & 111 & 20942.7 & 188.7 & 49 & 6311.1 & 128.8 & 28 & 1862.3 & 66.5 \\
\hline 9 & 109 & 20937.2 & 192.1 & 43 & 6348.8 & 147.6 & 21 & 2888.0 & 137.5 \\
\hline 10 & 114 & 22759.0 & 199.6 & 46 & 6120.5 & 133.1 & 22 & 1970.0 & 89.5 \\
\hline 1 & 125 & 22405.2 & 179.2 & 50 & 6336.7 & 126.7 & 23 & 2078.7 & 90.4 \\
\hline 2 & 133 & 23661.7 & 177.9 & 51 & 6118.4 & 120.0 & 30 & 2299.3 & 76.6 \\
\hline
\end{tabular}

Source: JCABC, 1794-1813.

des Canadiens anglais à Montréal au détriment des premiers (tableau XIII et graphique I). Cette différenciation très nette entre régions et ethnies, peut s'expliquer en partie par le fait qu'à Québec, capitale du Bas-Canada, certains postes officiels de l'administration sont par définition la propriété du groupe anglophone qui fait le lien avec la métropole, ou par son corollaire, à savoir que la structure des postes s'avère plus volatile à Montréal.

(5) Quelques tendances

Il est possible d'extraire des tableaux qui précèdent, certaines tendances qui percent dans divers secteurs et qui les lient ou les opposent à l'intérieur du grand brassage qui s'opère au tournant du XIX ${ }^{\mathbf{e}}$ siècle dans le Bas-Canada.

(a) Dans la fraction des emplois et des pensions réservée aux Canadiens français, la noblesse seigneuriale et militaire en accapare une portion importante en nombre et surtout en valeur. 
(b) Ensuite, tant pour les pensions que pour les salaires, le niveau moyen est en général beaucoup plus élevé pour les Canadiens anglais que pour les Canadiens français. Cependant, l'écart est exponentiel pour les pensions, plus doux pour les salaires.

(c) Contrairement au nombre de pensions, somme toute assez stable, celui des emplois croît rapidement. De plus, alors que les pensions consenties à des Canadiens français sont plus nombreuses que celles destinées aux Canadiens anglais pendant presque toute la période, la situation inverse prévaut en ce qui concerne les salaires en général et aussi chacune des catégories de salaire que nous avons découpées.

(d) Tant pour la masse des pensions dans le Bas-Canada que pour la moyenne des salaires à Montréal, on note un renversement radical dans les positions relatives des deux groupes ethniques au cours de la période.

(e) Il n'a pas été possible d'analyser la structure régionale des pensions. Quant aux salaires, la portion des emplois à Québec demeure aux environs de $60 \%$, celle de Montréal, autour de $25 \%$. Cependant, le rapport des salaires moyens est fort instable: ce qui fait croire qu'une série de forces plus ou moins différentes opèrent dans les deux régions.

Voilà, en style télégraphique, une synthèse de quelques-unes des tendances majeures qui ressortent de notre analyse. Ceci nous conduit à chercher, derrière ces tableaux simplificateurs, des mécanismes qui viendraient élucider la dynamique de ces ajustements différenciés. La prochaine section propose une hypothèse et la vérifie au moyen d'une série de tests statistiques.

\section{Deux types de patronage: un test statistique}

L'analyse quantitative de la dernière section a dévoilé l'existence de certaines divergences notables tant dans l'allocation des emplois et des pensions que dans le niveau des rémunérations et des paiements de transfert entre ethnies et

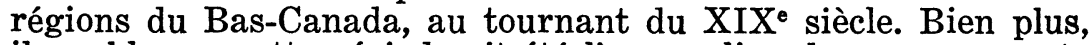
il semble que cette période ait été l'espace-lieu de renversements prononcés dans la distribution de la masse salariale et des pensions entre groupes et de par le territoire.

D'autre part, notre discussion antérieure avait présenté un cadre de référence et un tissu d'expériences qui montraient l'influence des valeurs et des priorités de l'Etat dans l'allocation et la distribution des biens publics, et l'importance qu'attachaient 
Nombre, somme et moyenne des salaires

à Québec et à Montréal - par groupe ethnique (1794-1812)

\begin{tabular}{|c|c|c|c|c|c|c|c|c|c|c|c|c|}
\hline & \multicolumn{6}{|c|}{ Québec } & \multicolumn{6}{|c|}{ Montréal } \\
\hline & & CF & & & $\mathrm{CA}$ & & & $\mathrm{CF}$ & & & $\mathrm{CA}$ & \\
\hline & $\begin{array}{c}\text { Nom- } \\
\text { bre }\end{array}$ & Total & Moyenne & $\begin{array}{l}\text { Nom- } \\
\text { bre }\end{array}$ & Total & Moyenne & $\begin{array}{l}\text { Nom- } \\
\text { bre }\end{array}$ & Total & Moyenne & $\begin{array}{c}\text { Nom- } \\
\text { bre }\end{array}$ & Total & Moyenne \\
\hline 1794 & 17 & 1477.0 & 86.8 & 44 & 9605.1 & 218.3 & 9 & 737.6 & 82.0 & 15 & 800.7 & 53.4 \\
\hline & 13 & 932.2 & 71.7 & 44 & 9086.8 & 206.5 & 11 & 1442.1 & 131.1 & 19 & 1689.5 & 88.9 \\
\hline 6 & 16 & 1559.8 & 97.5 & 47 & 10829.6 & 230.4 & 9 & 1650.0 & 183.3 & 19 & 2505.4 & 131.9 \\
\hline 7 & 19 & 1534.9 & 80.8 & 47 & 10539.8 & 224.3 & 9 & 1700.0 & 188.9 & 22 & 2979.3 & 135.4 \\
\hline 8 & 16 & 1752.2 & 109.5 & 45 & 11030.0 & 245.1 & 10 & 1749.6 & 175.0 & 20 & 2821.9 & 141.1 \\
\hline 9 & 18 & 1788.0 & 99.3 & 50 & 12139.5 & 242.8 & 9 & 1700.0 & 188.9 & 20 & 2393.5 & 144.7 \\
\hline 1800 & 18 & 2072.6 & 115.1 & 47 & 14588.1 & 310.4 & 9 & 1723.3 & 191.5 & 27 & 3101.0 & 114.9 \\
\hline 1 & 19 & 2443.0 & 128.6 & 50 & 14675.7 & 293.5 & 10 & 2000.0 & 200.0 & 21 & 3474.9 & 165.5 \\
\hline 2 & 21 & 2667.2 & 127.0 & 53 & 15319.8 & 289.1 & 9 & 1950.0 & 216.7 & 22 & 2653.4 & 120.6 \\
\hline 3 & 26 & 2652.3 & 102.0 & 50 & 14650.5 & 293.0 & 7 & 2069.6 & 295.7 & 23 & 3851.2 & 167.4 \\
\hline 4 & 32 & 3128.1 & 97.8 & 45 & 14570.7 & 323.8 & 9 & 1992.3 & 221.4 & 24 & 3861.2 & 160.9 \\
\hline 5 & 32 & 3292.4 & 102.9 & 59 & 13991.0 & 237.1 & 13 & 2170.6 & 167.0 & 23 & 3926.6 & 170.7 \\
\hline 6 & $3 \overline{6}$ & 3697.0 & 102.7 & 55 & 13520.9 & 245.8 & 16 & 2151.8 & 134.5 & 24 & 5476.7 & 228.2 \\
\hline 7 & 34 & 4232.8 & 124.5 & 64 & 28854.0 & 357.1 & 16 & 1367.4 & 85.5 & 28 & 3884.4 & 138.7 \\
\hline 8 & 39 & 4992.6 & 128.0 & 70 & 15883.1 & 226.9 & 19 & 1815.9 & 95.6 & 29 & 4468.2 & 154.1 \\
\hline 9 & 38 & 4119.2 & 111.3 & 70 & 16751.0 & 239.3 & 16 & 2093.0 & 130.8 & 26 & 4228.8 & 162.6 \\
\hline 1810 & 43 & 5037.5 & 117.2 & 69 & 17654.5 & 255.9 & 17 & 1735.4 & 102.1 & 28 & 4358.1 & 155.6 \\
\hline 1 & 51 & 5375.6 & 105.4 & 72 & 16962.6 & 235.6 & 16 & 1843.0 & 115.2 & 32 & 4466.9 & 139.6 \\
\hline 2 & 54 & 5015.1 & 92.9 & 76 & 18553.7 & 244.1 & 16 & 1724.6 & 107.8 & 34 & 4366.8 & 128.4 \\
\hline
\end{tabular}

Source: JCABC, 1794-1813. 
les diverses strates de la population à une part aux places. Ce qui nous a conduit à formuler l'hypothèse qu'il $\mathrm{y}$ a eu patronage dans le Bas-Canada au tournant du $\mathrm{XIX}^{\mathrm{e}}$ siècle et que les disparités observées en section $V$ résultent d'un processus de patronage qu'il nous reste à préciser.

Dans la présente section, nous formulerons un modèle très simple qui spécifie les voies et modes d'opération du patronage. La confrontation du modèle avec les données fort détaillées que nous avons accumulées permettra d'en jauger la justesse. Celle-ci se mesure en effet à l'efficacité avec laquelle le modèle "explique" les comportements observés, comportements divers qui se concrétisent en ces tendances multiples que nous avons relevées plus haut.

\section{(1) Un modèle plausible}

Le patronage est un phénomène complexe qu'on a étudié sous ses aspects économiques, politiques et anthropologiques. ${ }^{172}$ Aux fins de notre analyse, nous le définirons comme l'une des voies utilisées par l'Etat, via ses agents au pouvoir, pour dispenser ses faveurs en fonction de certaines loyautés qu'il juge opportunes ou utiles. Il s'agit donc d'une forme de paiement pour des services intangibles ou du moins implicites. Si, de prime abord, elle peut sembler un don, elle n'en constitue pas moins une forme d'échange où les contreparties sont plus ou moins explicitement disjointes.

Nous nous intéressons moins ici aux motivations qui animent ce procès qu'aux méthodes qu'il développe. Ainsi, l'on peut reconnaître deux modes fort différents de patronage qui permettent d'ores et déjà une spécification de méthodes diverses. D'une part, le patronage peut s'effectuer par une répartition inégale des emplois ou des pensions qui privilég1e certains groupes ou régions. Nous nommerons ce procédé patronage de répartition (type I). D'autre part, le patronage peut aussi emprunter la voie d'une répartition proportionnelle des emplois et des pensions, simplement par l'octroi, à certains groupes ou à certaines régions, de niveaux de rémunération ou de pension plus élevés que ceux payés ailleurs ou à d'autres groupes. Dans ce cas, il s'agit du patronage de rétribution (type II).

Si nous postulons un cas-limite où le procédé est strictement du type I (patronage de répartition), on en décèlera la présence en comparant les probabilités de décrocher une pension ou un emploi selon la nature des groupes. On s'attendra alors à des

172 Voir note 56. 
probabilités fort différentes de pourcentages élaborés à partir d'une représentation proportionnelle. Mais si au contraire, nous partons de l'autre cas-limite, celui où le procédé est strictement du type II (patronage de rétribution), on en détectera la présence en confrontant les niveaux de salaires et de pensions des divers groupes.

Bien sûr, ces procédés peuvent se combiner selon des dosages divers, tous plausibles. Le patronage concret mêle l'un et l'autre procédés en une potion spécifique mieux ajustée aux mœurs et aux besoins. En fait, les institutions que se donne le patronage constituent en quelque sorte la solution concrète à ce problème du choix d'un mélange optimal. Choix qui obéit, en fin de compte, à diverses contraintes: les besoins collectifs qu'il faut satisfaire, la sensibilité des populations à l'un ou l'autre procédé, toute une série de facteurs sociologiques que nous ne pouvons discuter ici.

La liste civile du Bas-Canada au tournant du XIX $\mathrm{XIècle}^{\mathrm{e}}$ s'avère une forme concrète de patronage. A ce titre, on devrait pouvoir la dissoudre en ces deux formes que nous avons définies plus haut. Cependant, à cause des divergences significatives entre le monde des pensions et celui des salaires, il nous a paru plus utile de dissocier ces deux canaux du patronage pour en distiller séparément les composantes. Dans chaque cas, nous chercherons, dans les mixtions différentes des deux ingrédients, la raison des tendances diverses que nous avons notées dans l'un et l'autre cas.

\section{(2) Un test statistique grossier du modèle}

Pour repérer la présence de l'un ou l'autre type de patronage et déterminer la nature différenciée de leur opération dans le monde des pensions et celui de la fonction publique, nous avons appliqué certaines techniques statistiques qui permettent de mesurer les effets composés sur une variable dite dépendante de variables numériques et d'attributs ou catégories dites variables indépendantes. ${ }^{173}$ Aux fins de la présente étude, nous nous limiterons à l'examen de relations linéaires fort simples entre ces variables. Ces relations seront spécifiées grâce à une technique élémentaire dite analyse de régression. Sans nous étendre ici sur des problèmes méthodologiques sur lesquels nous reviendrons en appendice, disons que cette technique permet de mesurer

${ }^{173}$ On dit qu'une variable $Y$ est fonction d'une variable $X$ ou dépend d'une variable $X$ quand une connaissance de $X$ permet de prédire la valeur de Y. On nomme variables indépendantes celles qui aident à prédire et variables dépendantes celles qui sont prédites. 
la dimension des coefficients de la relation linéaire qui soude les variables indépendantes à la variable dépendante qui nous intéresse.

Dans un premier temps, nous nous attacherons à calculer la valeur des coefficients $b_{0}, b_{1}$, etc. dans des relations linéaires de la forme suivante:

$$
\begin{aligned}
& P\left(G_{i}\right)=b_{0}+b_{1} X_{1}+b_{2} x_{2}+\ldots+b_{n} X_{n} \\
& \text { où } P\left(G_{i}\right)=\text { probabilité de faire partie du groupe } i \\
& \text { (variable dépendante) } \\
& \mathrm{X}_{1} \ldots \mathrm{X}_{\mathrm{n}}=\text { une série de } \mathrm{n} \text { variables indépendantes qui } \\
& \text { sont censées affecter cette probabilité } \\
& P\left(G_{i}\right) \\
& b_{0} \ldots b_{n}=\text { coefficients qui relient les variables indé- } \\
& \text { pendantes à la variable dépendante et } \\
& \text { doivent être définis à partir des données. }
\end{aligned}
$$

Ainsi, nous pourrons nous interroger sur la probabilité d'être canadien-français $\left[P\left(G_{c f}\right)\right]$ si nous spécifions l'année $\left(\mathrm{X}_{1}\right)$ et le niveau du salaire $\left(\mathrm{X}_{2}\right)$. Dans ce cas, l'équation prendra la forme

$$
P\left(G_{c f}\right)=b_{0}+b_{1} X_{1}+b_{2} X_{2}
$$

et pour autant que nous pouvons estimer la grandeur des coefficients $b_{0}, b_{1}$ et $b_{2}$, il deviendra possible de prédire la probabilité d'être canadien-français pour le cas d'un salaire de $£ 300$ versé en 1805 par exemple.

Dans un deuxième temps, nous nous attacherons à supputer la valeur des coefficients $c_{0}, c_{1} \ldots$ etc. dans des relations linéaires de la forme suivante:

$$
\text { où } \begin{aligned}
& L_{j}=c_{0}+c_{1} X_{1}+c_{2} X_{2}+\ldots+c_{n} X_{n} \\
& L_{j}=\text { niveau de la rétribution (salaire ou pension) } \\
& X_{1} \ldots X_{n}=\begin{array}{l}
\text { une série de n variables indépendantes qui } \\
\text { supposément affectent ce niveau } L_{j}
\end{array} \\
& c_{0} \ldots c_{n}=\begin{array}{l}
\text { coefficients qui relient les variables indé- } \\
\text { pendantes à la variable dépendante et } \\
\text { doivent être définis à partir des données. }
\end{array}
\end{aligned}
$$

Ainsi, nous pourrons nous demander quel est le niveau prévisible d'une pension $\left(\mathrm{L}_{\mathrm{p}}\right)$ si nous précisons qu'il s'agit d'un Canadien français $\left(\mathrm{X}_{1}\right)$ et de l'année $1805\left(\mathrm{X}_{2}\right) .174$

174 On pourra se surprendre de voir un attribut comme l'ethnicité présenté sous forme de variable numérique. Il s'agit là d'un procédé fort utile par lequel une variable dichotomique (blanc-noir, recto-verso, guerre-paix) est représentée par la dichotomie $0-1$. On nomme une variable dichotomique de ce type, variable fictive. Nous reviendrons sur le sujet en appendice. 
En calculant les coefficients d'une série de droites de régression des deux types (1) et (3) tant pour les pensions que pour les salaires, nous avons pu définir précisément des relations qui nous permettent une prédiction des probabilités et des niveaux observés. Ce qui plus est, il est possible de déterminer si ces équations ajustées aux données constituent des approximations valables de la réalité. Par une série de tests dont nous dirons un mot en Appendice $B$, on peut en effet préciser la validité des coefficients estimés et la justesse des équations pour ce qui est de l'image qu'elles construisent de la réalité.

Ainsi que nous le verrons, ces tests statistiques nous ont permis de mettre à jour les mécanismes fort différents qui soustendent les mondes des pensions et de la fonction publique comme instruments de patronage.

\section{(3) Les pensions}

Déjà, dans la section $\mathrm{V}$, les données provenant des Journaux de la Chambre d'Assemblée ont révélé une stabilité assez surprenante de la structure ethnique du nombre des pensions, encore que la structure des sommes allouées aux divers groupes ethniques change de façon dramatique au cours de la période (tableau IX). Nous savons aussi que le repérage de la structure régionale de ces transferts de fonds n'a pu être établi de façon nette, à cause de l'imprécision des données.

Dans la présente section, nous voudrons aller derrière les phénomènes notés au tableau IX, pour mettre à jour les mécanismes qui sous-tendent ce renversement.

(i) Effet de répartition

Même si les Canadiens français ne sont jamais moins des neuf-dixièmes de la population du Bas-Canada au cours des années 1794-1812, ils n'accaparent jamais plus de $82 \%$ des pensions. Ce plateau, autour des $80 \%$, dure plus de dix ans: de 1794 jusqu'en 1805. Là s'amorce un déclin que vient précipiter, en 1810 , un changement dans les politiques de pensions.

S'il y a patronage de répartition dans le cas des pensions, nous pouvons faire l'hypothèse suivante: à mesure que le niveau des pensions augmente - car on réserve aussi les meilleures places à ses amis - et à mesure que le temps passe, la probabilité d'être un récipiendaire canadien-français décroît. Nous avons justement voulu vérifier si tel est le cas. Les équations (4) et (5) du tableau XIV mesurent l'effet linéaire du niveau des pensions sur la probabilité du récipiendaire d'être canadien-français, et l'effet linéaire conjugué de l'année ou du temps et du niveau des pensions sur cette même probabilité. 
TABLEAU XIV

Quelques résultats de l'analyse de régression linéaire pour le secteur des pensions

I. - Effet de répartition ethnique

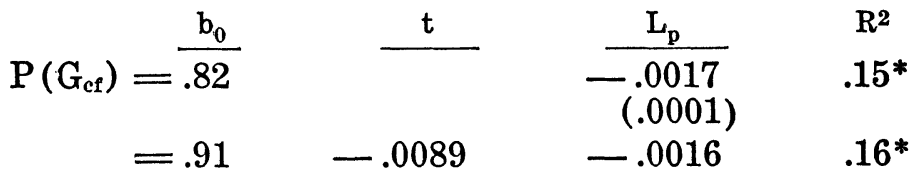

$$
\begin{aligned}
& \text { (.0027) (.0001) }
\end{aligned}
$$

II. - Effet de rétribution

$$
\begin{aligned}
& \mathrm{L}_{\mathrm{p}}=\frac{\mathrm{c}_{0}}{23.03} \quad \frac{\mathrm{t}}{+.19} \quad \frac{\mathrm{A}}{(.65)} \quad \frac{\mathrm{tA}}{+\underset{(.56)}{7.59}} \mathrm{R}^{2} \\
& \begin{array}{lrrrr}
=27.37 & -.16 & -17.75 & +8.86 & .21^{*}
\end{array}
\end{aligned}
$$

Les résultats avalisent l'hypothèse: les coefficients négatifs des variables indépendantes prouvent qu'à mesure que le niveau de pension croît et que nous progressons dans la période, la probabilité d'être un récipiendaire canadien-français diminue. Ainsi, selon l'équation (4), la probabilité d'être un récipiendaire canadien-français passe de $80.3 \%$ pour une pension de $£ 10$ à $65 \%$ pour une pension de $£ 100$. Suivant la même logique, la probabilité d'être canadien-français s'il s'agit d'une pension de $£ 100$ en 1802 (la dixième année), ${ }^{175}$ est selon l'équation (5) de $66.1 \%$, alors que pour la même somme en 1807 (la quinzième année), la probabilité descend à $61.65 \%$.

Il est possible de soumettre ces équations en général et chacun des coefficients des variables indépendantes à des tests qui établissent le degré de confiance qu'on peut avoir en la justesse de l'équation, comme appareil de prédiction, et dans celle des coefficients évalués, comme mesure de l'importance de la contribution de la variable indépendante à l" "explication" de la variable dépendante. Le résultat de ces tests se trouve aussi dans le tableau XIV. Le coefficient de détermination $\left(R^{2}\right)$ mesure la justesse de l'équation comme tout, i.e. mesure la proportion de la variation dans la variable dépendante qui est attribuable au modèle linéaire composé des variables indépendantes retenues. Nous avons pris l'habitude d'indiquer par une

\footnotetext{
175 Tous les calculs ont été faits à partir de $1793=1,1794=2$, etc.
} 
astérisque les équations qui satisfont le test. ${ }^{176}$ En ce qui a trait aux coefficients des variables indépendantes, il est possible d'en vérifier la signification en en comparant la grandeur avec l'erreur-type qui y correspond. L'erreur-type est l'écart-type de la distribution de toutes les évaluations possibles du coefficient; et pour fixer les idées, il suffit de retenir que le coefficient peut être considéré comme significatif au niveau de 5\% (voir note 176) s'il est au moins deux fois plus grand que son erreurtype. Pour faciliter ce calcul, nous avons inséré l'erreur-type de chaque coefficient entre parenthèses en dessous de chaque coefficient. ${ }^{177}$

Dans le cas des équations (4) et (5), la grandeur des $R^{2}$ et des erreurs-types indique que les deux équations et les trois coefficients passent le test de signification au niveau de $5 \%$. Il semble donc clair qu'à mesure que les niveaux de pension grimpent et que la période s'écoule, la probabilité d'être un récipiendaire de pension canadien-français s'amoindrit - ainsi que nous en avions fait l'hypothèse. Il $\mathrm{y}$ a donc eu patronage de répartition ethnique dans la période qui nous intéresse.

(ii) Effet de rétribution

Non seulement peut-on affirmer qu'il y a eu patronage de type I. De bonnes raisons portent à croire qu'il s'y est mêlé du

${ }^{176}$ Le lecteur devra se reporter à notre appendice pour une discussion plus élaborée de ces tests. Cependant, on peut signaler le sens de ces tests en quelques mots. Le coefficient $R^{2}$ mesure le degré de covariation entre les valeurs de la variable dépendante observée effectivement et celles calculées par le truchement de la droite de régression, i.e. à partir d'une connaissance des variables indépendantes seulement. Nous dirons que la droite de régression est significative au niveau de $5 \%$ s'il y a moins de 5 chances sur 100 que l'on ait une covariation comme celle que nous avons obtenue à partir d'une hypothèse que la variable dépendante est indépendante du système linéaire de variables indépendantes considéré. Comme nous avons plus de 840 observations dans notre étude sur les pensions, la valeur critique est autour de .01; mais on pourra vérifier plus précisément dans chaque cas dans un quelconque manuel de statistiques. Voir, par exemple, G. W. Snedecor, Statistical Methods (Ames, Iowa, 1946), 351.

177 Nous avons décrit dans le corps du texte le test conventionnel. Comme nous avons ajusté la droite de régression aux données par l'une des méthodes traditionnelles - la méthode des moindres carrés -, il faut bien accepter que la droite que nous avons calculée n'est pas nécessairement la seule que nous ayons pu tirer des données. L'erreur-type mesure le degré d'imprécision de nos évaluations des coefficients. Il constitue en quelque sorte un coefficient d'incertitude quant à la précision de l'estimation. Or le test laisse croire que l'on peut considérer le coefficient comme significatif au niveau de 5\% (voir note 176) si l'erreur-type est moins de 50\% du coefficient. Pour une discussion simple et fort claire de ces problèmes, le lecteur peut consulter D. B. Suits, Statistics: An Introduction to Quantitative Economic Research (Chicago, 1963), chap. 7. 
GRAPHIQUE 1

MOYENNE DES SALAIRES DES CANADIENS FRANÇAIS

ET DES CANADIENS ANGLAIS À MONTRÉAL

(1794-1812)

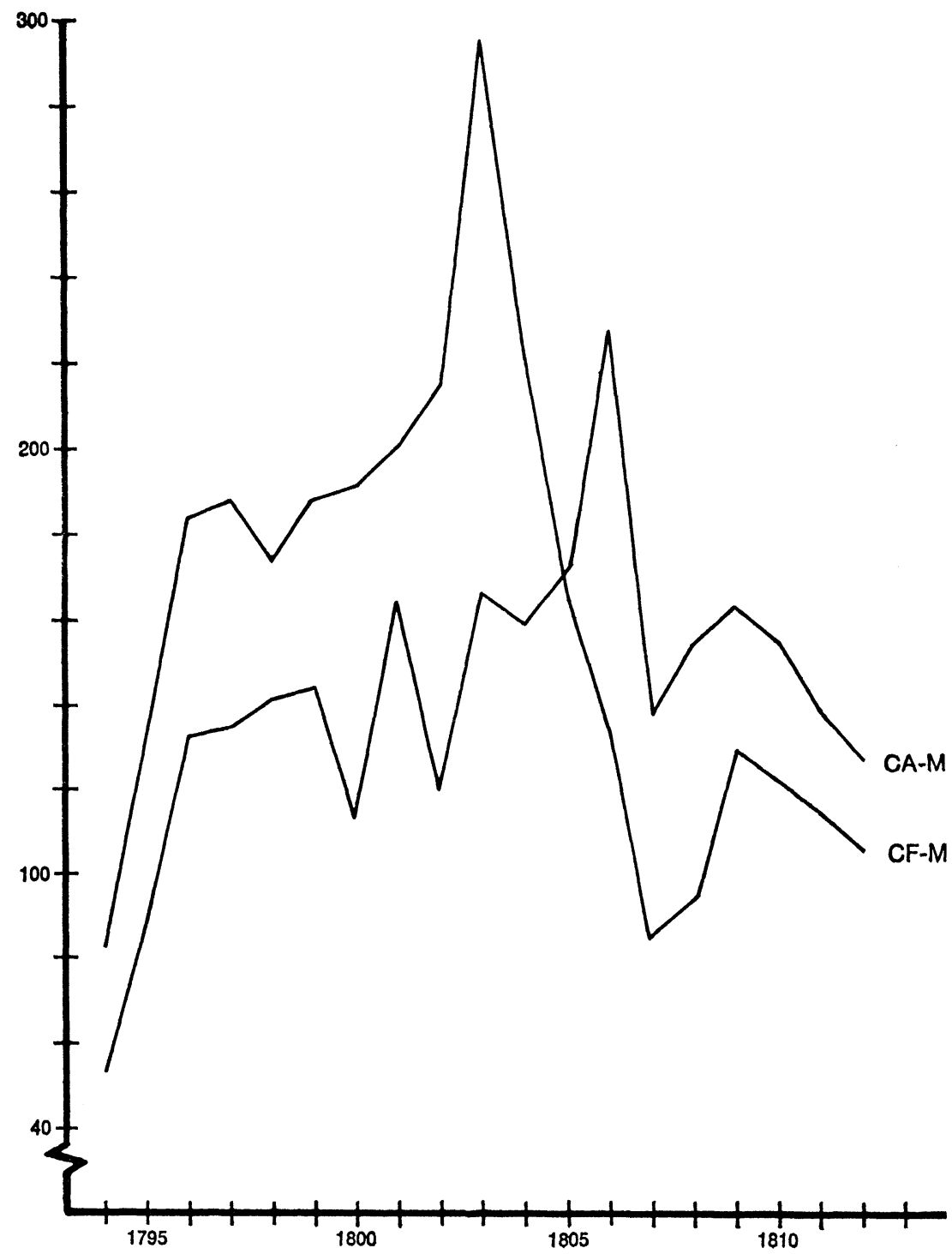

Source : Tableau XIII 
GRAPHIQUE 2

NIVEAU MOYEN OBSERVÉ ET PRÉDIT DES PENSIONS PAR ETHNIE

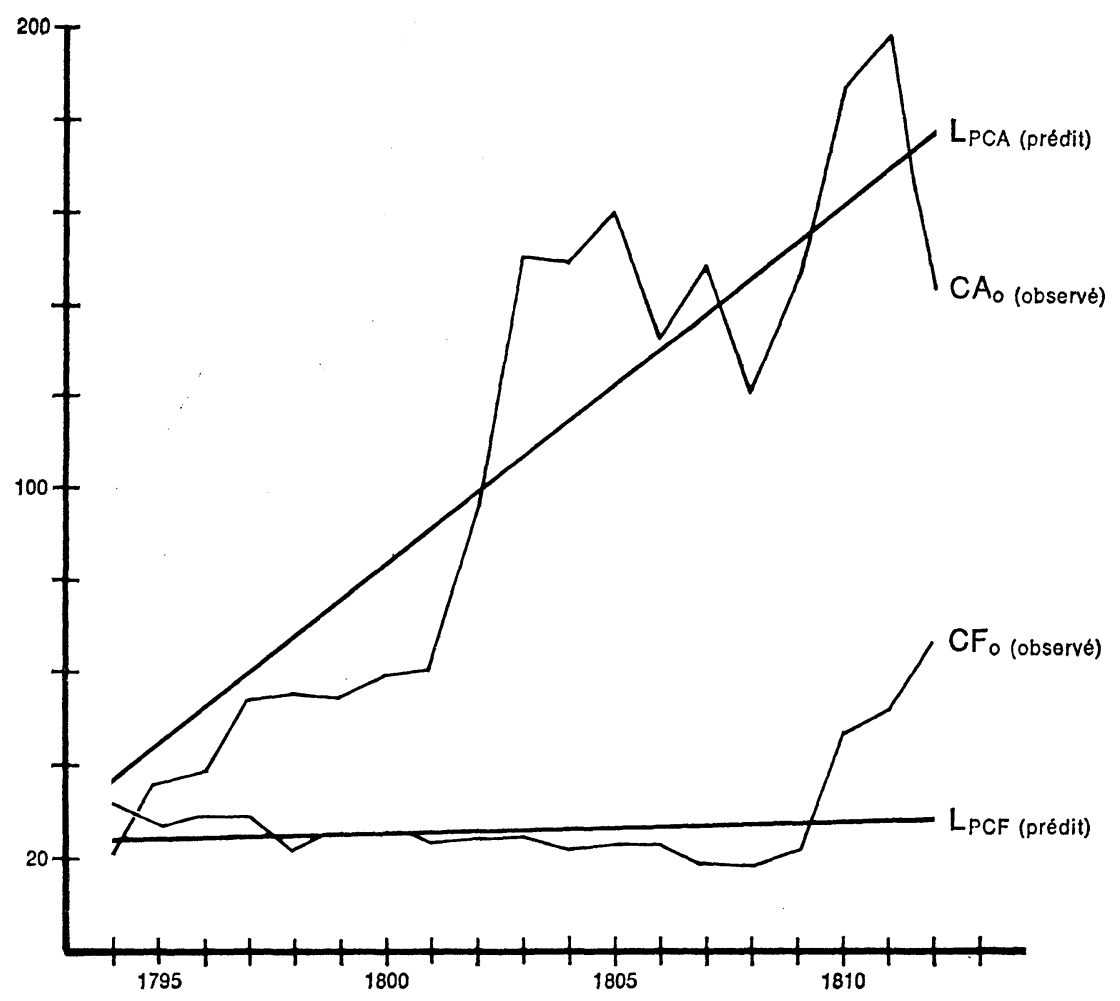

Source: Tableau IX et équation 6 
patronage de rétribution. En effet, un examen rapide du graphique II fait voir l'écart grandissant entre la moyenne des pensions payées aux récipiendaires des deux groupes ethniques. Nous avons tenté de prendre une mesure plus exacte de cet effet de rétribution.

Afin de spécifier statistiquement cet écart, nous avons eu recours à deux équations: l'une introduisant comme variable indépendante l'effet conjugué du temps et de l'ethnie (équation 6) ; l'autre introduisant en sus de cet effet conjugué, une variable dichotomique (anglais, non-anglais) qui affecte le niveau de la droite de régression plutôt que la pente (équation 7). ${ }^{178}$ L'addition de cette variable dichotomique n'amplifie

$$
Y=a_{0}+a_{1} X+a_{3} Z
$$

Si $Z=0, Y=a_{0}+a_{1} X$

Si $Z=1, Y=a_{0}+a_{1} X+a_{3}=\left(a_{0}+a_{3}\right)+a_{1} X$

Dans ce cas, l'inclusion de la variable fictive $Z$ a laissé la pente de la droite dans l'espace YX inchangée; mais elle a donné naissance à deux niveaux différents selon la valeur de $\mathrm{Z}$. D'autre part, la variable fictive peut être conjuguée à une autre variable indépendante, numérique celle-là. Dans ce cas, la droite de régression engendre deux lignes de régression à pente différente selon la valeur de Z. Symboliquement, on écrira:

$$
Y=a_{0}+a_{1} X+a_{2} X Z
$$

Si $Z=0, Y=a_{0}+a_{1} X$

Si $Z=1, Y=a_{0}+a_{1} X+a_{2} X=a_{0}+\left(a_{1}+a_{2}\right) X$

Dans ce cas, la variable fictive change la pente de la droite dans l'espace $Y X$. Il est évidemment possible d'utiliser les deux procédés en même temps. Ceci permet de changer le niveau et la pente de la droite de régression selon la valeur de $Z$. Dans l'équation (6), la variable anglais, non-anglais est jumelée à la variable numérique temps, alors que dans l'équation (7), la variable fictive anglais, non-anglais est utilisée à la fois isolément et en conjonction avec la variable numérique temps. Pour une discussion simple de ce problème, voir J. Johnston, Econometric Methods (New York, 1963), $221 s q q$.

pas le pouvoir d'explication du modèle - les deux valeurs $R^{2}$ sont les mêmes - ; et comme son coefficient n'est pas significatif au niveau de $5 \%$, nous n'avons retenu que l'équation (6) dans l'analyse qui suit.

178 On peut manier de bien des façons les variables fictives comme variables indépendantes dans un modèle linéaire. D'une part, on peut utiliser la variable fictive isolément comme variable indépendante ordinaire. Puisque la variable fictive revêt seulement deux valeurs (0 et 1$)$, le coefficient de la variable fictive devient un nombre qui sera multiplié par 0 ou par 1 selon le cas. Ce coefficient multiplié par 0 s'annule; multiplié par 1 , il s'additionne naturellement au terme constant de l'équation. Symboliquement, si $\mathrm{Y}$ est la variable dépendante et si $X$ est une variable fictive indépendante, on peut désomposer les résultats de notre analyse de régression comme suit: 
L'équation (6) utilise la variable fictive anglais-nonanglais en conjonction avec la variable numérique temps, de façon à mesurer l'écart des pentes qui s'ajustent aux données pour les niveaux de rétribution des deux groupes ethniques. Partant de l'équation (6)

$$
\mathrm{L}_{\mathrm{p}}=23.03+.19 \mathrm{t}+7.59 \mathrm{tA}
$$

nous voyons que si $A=0$ (i.e. s'il s'agit de Canadiens nonanglais), l'équation devient

$$
\mathrm{L}_{\text {pcf }}=23.03+.19 \mathrm{t}
$$

Si $A=1$ (i.e. s'il s'agit de Canadiens anglais), l'équation devient

$$
\begin{aligned}
\mathrm{L}_{\text {pca }} & =23.03+(.19+7.59) \mathrm{t} \\
& =23.03+7.78 \mathrm{t}
\end{aligned}
$$

Ces deux droites ont été surimposées sur le graphique des niveaux moyens de pension (graphique II).

Le graphique II confirme visuellement ce que nous pouvions déjà lire dans l'équation (6), i.e. qu'il se produit un effet de rétribution très important lié au temps. Il $\mathrm{y}$ a donc patronage de rétribution dans le secteur des pensions et celui-ci s'accroît de façon dramatique avec le passage du temps. Ce graphique suggère aussi que l'on pourra améliorer le modèle en dépassant le simplisme des relations linéaires. Ainsi, une relation de type exponentiel cernera de plus près le sentier des niveaux moyens de pensions des Canadiens anglais. Toutefois, nous ne procéderons à aucune démarche de ce type dans le présent article. ${ }^{179}$

Nos résultats sont donc bien clairs. Il y a patronage de répartition et de rétribution dans le secteur des pensions; l'une et l'autre composantes ont sculpté, par leur impact significatif, le paysage des paiements de transferts au tournant du XIX ${ }^{\circ}$ siècle. Nous avons pu découvrir que la probabilité d'être un pensionné canadien-français s'amenuise à mesure que les années passent et que l'on escalade l'échelle des pensions (niveaux). En ce qui concerne ces niveaux, nous avons pu établir que le niveau moyen demeure fort stable pour les Canadiens français, alors qu'il grimpe rapidement pour les Canadiens anglais tout au cours de la période.

\section{(4) Les salaires}

$\mathrm{Du}$ côté de la fonction publique, les résultats de la section $\mathrm{V}$ révèlent des disparités étonnantes entre les régions et les groupes

179 Pour plus de détails, voir F. C. Mills, Statistical Methods (New York, 1955), appendice F. 
GRAPHIQUE 3

NIVEAU MOYEN OBSERVÉ ET PRÉDIT DES SALAIRES PAR ETHNIE

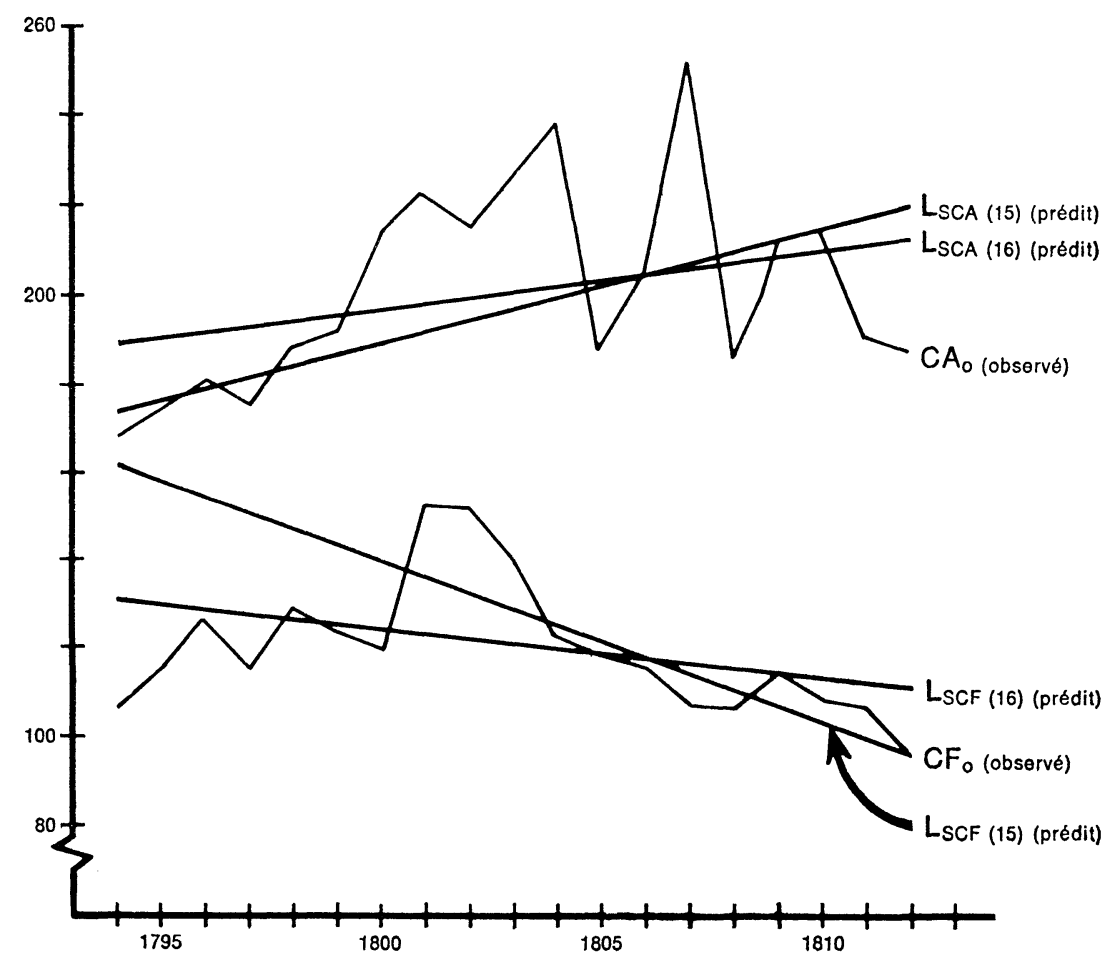

Source: Tableau XI, équations 15 et 16 
GRAPHIQUE 4
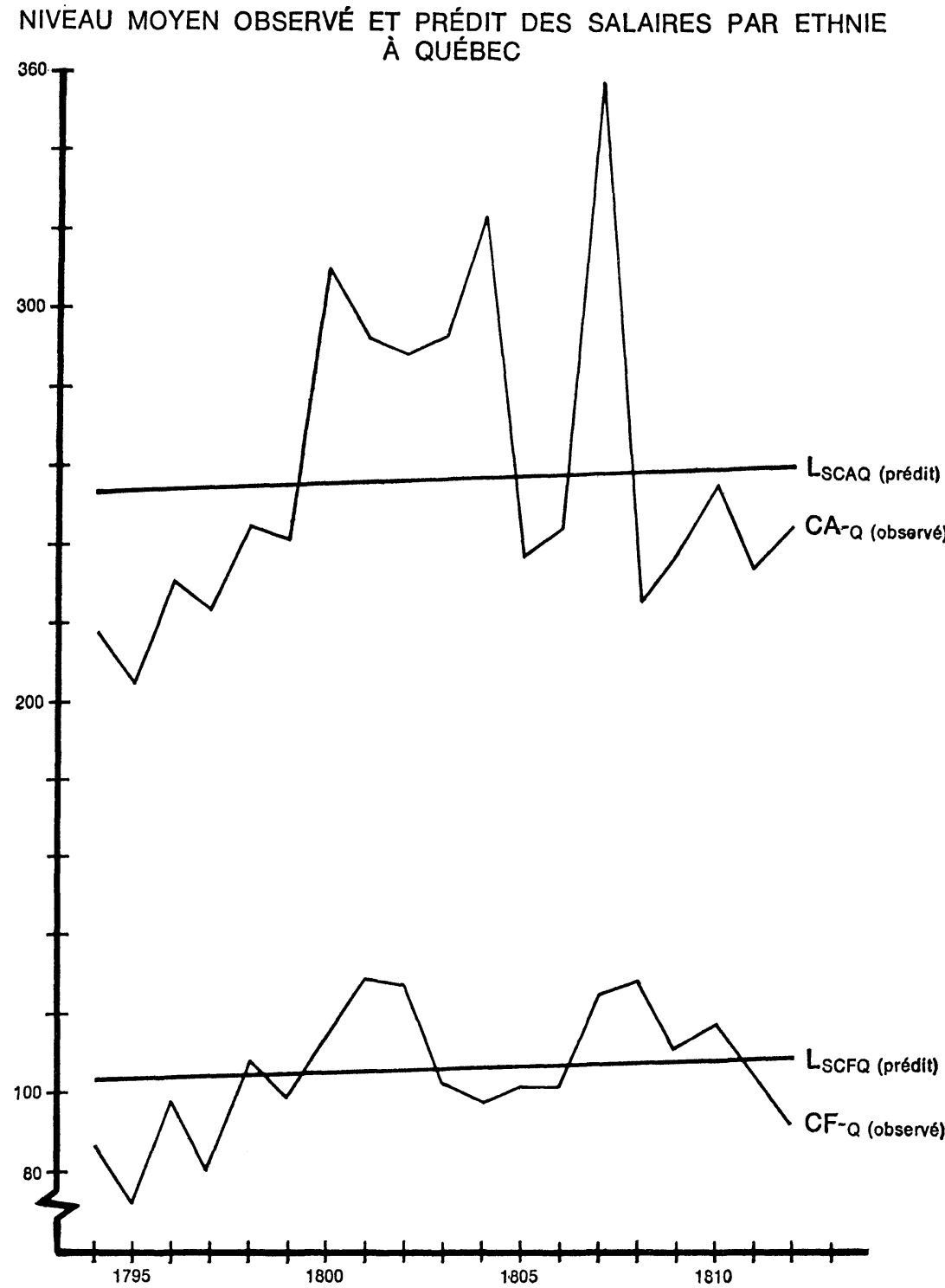

Source : Tableau XII et équation 17 
ethniques. Il faut donc examiner de plus près les tendances recélées dans les tableaux X, XI, XII et XIII.

\section{(i) Effet de répartition}

Ainsi que nous le mentionnions antérieurement, la fonction publique se gonfle avec la population du Bas-Canada. Jamais, pourtant, les Canadiens français ne pourront occuper plus de $40 \%$ des postes et toucher plus de $25 \%$ de la masse salariale. Bien plus, ce groupe ethnique est minoritaire à tous les niveaux de salaires. De même, nous avons noté une structure régionale des emplois assez stable. Bien que les villes de Québec et de Montréal soient de même dimension vers 1806-1808, le nombre de postes à Québec atteint au moins le double de celui des places à Montréal tout au long de la période. ${ }^{180}$

Nous avons donc été amenés à formuler l'hypothèse de l'existence du patronage de répartition. Aux fins de comparaison, nous avons employé les équations (8) et (9) qui sont, par leur forme, identiques aux équations (4) et (5) que nous avons calculées pour les pensions. A cause de la meilleure qualité des données, nous avons aussi pu ajouter un effet géographique sur la probabilité d'être canadien-français dans l'équation (10).

Les résultats de ce test du patronage de répartition sont présentés au tableau XV (I). On y voit que la probabilité d'être canadien-français fléchit à mesure que le salaire croît (équation 8), mais que l'effet additionné du temps et de la grandeur du salaire est plus composite. En effet, la probabilité d'être canadien-français augmente à mesure que la période s'écoule, même si elle décline à mesure que le niveau du salaire s'élève (équation 9). Ainsi la probabilité qu'un Canadien français émarge au budget pour un poste de $£ 100$, en 1797 , est de l'ordre de $28.2 \%$, alors que la même probabilité pour le même poste est de $35.2 \%$ en $1807 .{ }^{181}$

Nous avons additionné un effet géographique à cette étude du patronage de répartition ethnique. La variable retenue est une variable fictive qui s'annule si le poste ne se trouve ni à Québec ni à Montréal. On peut voir dans l'équation (10) que si le poste de $£ 100$, dont il était question dans l'exemple du dernier paragraphe, est situé à Québec ou à Montréal, la probabilité que le détenteur en soit canadien-français s'accroît de $4.5 \%$.

180 Lambert parle de 12,000 habitants dans chaque ville en 1808 (op. cit., 1: 276; 2: 69). Pour sa part, Gray parle de 10,000 en se reportant aux années 1806-1808 (Letters from Canada [...], 56, 146).

181 On se souvient que dans le cas des pensions (équation 5), les deux effets étaient négatifs. 
Comme pour les pensions, on peut confirmer la justesse des équations et le degré de signification des coefficients. Nous disposons ici de plus de 2790 observations et la valeur critique de $R^{2}$ se situe entre .005 et .01, tandis que les coefficients doivent atteindre le double de leur erreur-type pour être significatifs au niveau de $5 \% .182$

\section{TABLEAU XV}

Quelques résultats de l'analyse de régression linéaire pour le secteur des salaires

PATRONAGE DE RÉPARTITION

I. - Effet de répartition ethnique

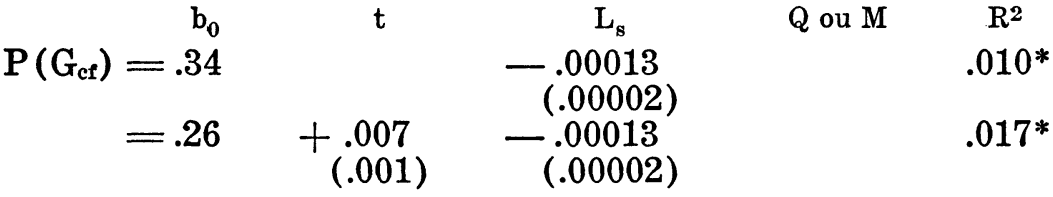

$$
\begin{aligned}
& =.22 \quad+\begin{array}{llll}
. .007 & -.00013 \\
(.001) & (.00002) & +.045 & .018^{*}
\end{array}
\end{aligned}
$$

II. - Effet de répartition géographique

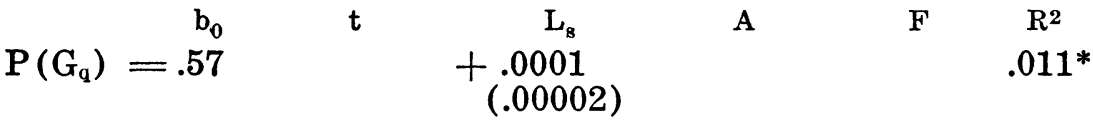

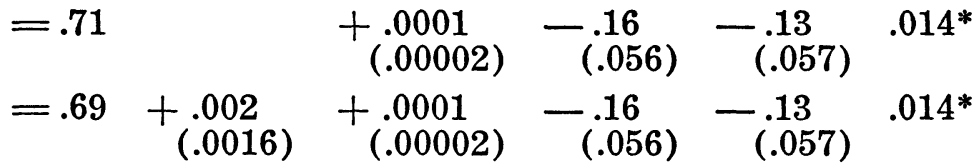

Nos données précises sur la localisation des détenteurs de postes nous ont permis d'examiner l'effet de répartition géographique. Nous pouvons conclure, à partir des résultats présentés au tableau XV (II), que la probabilité qu'un poste soit à Québec, s'accroît à mesure que le niveau de salaire s'élève (équation 11), qu'elle augmente avec le temps (équation 13) et qu'elle est plus forte pour les Canadiens français que pour les Canadiens anglais de quelque $3 \%$ pour un niveau de salaire et une année donnés (équation 12). 
(ii) Effet de rétribution

Si l'on peut capter des traces de patronage de type I, on en trouve aussi de patronage de rétribution. Un coup d'œil rapide sur le graphique III révèle des sentiers fort différents pour les niveaux moyens de salaire des détenteurs de postes des deux groupes ethniques. Pour pincer d'un peu plus près la mesure de cet effet de rétribution, nous avons procédé à peu près comme dans le cas des pensions. En effet, nous avons spécifié trois formes de relations linéaires: l'une comprend les variables fictives anglais, non-anglais et français, non-français isolément (tableau XVI, équation 14); la seconde conjugue les mêmes variables fictives avec la variable numérique temps (équation 15) ; la troisième utilise les variables fictives à la fois isolément et en conjonction avec la variable temps (équation 16).

Aux fins de comparaison, nous avons surajouté au graphique III les droites définies pour chaque groupe ethnique par les équations 15 et 16 . La première équation engendre des droites de régression à pentes différentes alors que la seconde engendre des droites de régression qui combinent les différences de pentes et de niveaux. Les deux formes de la relation ont le même coefficient $R^{2}$, mais les coefficients de l'équation (15) passent mieux l'épreuve des tests de signification. ${ }^{183}$

Bien que ni l'une ni l'autre des formes spécifiées par les équations 15 et 16 ne soient complètement satisfaisantes, nous préférons, malgré les raisons mentionnées au paragraphe précédent, les formes dérivées de l'équation (16). En effet, il semble que c'est le caractère linéaire du modèle qui nous crée des difficultés. Un examen attentif du graphique III révèle à la fois un effet de niveau et un effet de pente, mais aussi une ondulation qui culmine autour de 1802 pour la moyenne des salaires à des Canadiens français, et plus tard, pour la moyenne des salaires à des Canadiens anglais. Une relation linéaire ne saurait rendre un compte précis de ces sentiers ondulatoires.

${ }^{183}$ Le procédé de dérivation des équations des droites du graphique est le même que nous avons introduit en note 174 et utilisé en section (3) (ii) :

(i) $\mathrm{L}_{\mathrm{s}}=169.08-9.28 \mathrm{t}+11.81 \mathrm{tA}+5.60 \mathrm{tF}$

$\mathrm{Si} \quad \mathrm{A}=0$ et $\mathrm{F}=1$

$\mathrm{L}_{\mathrm{sct}}=169.08-3.68 \mathrm{t}$

Si $\quad \mathrm{F} \equiv 0$ et $\mathrm{A}=1$

$\mathrm{L}_{\mathrm{sca}}=169.08+2.51 \mathrm{t}$

(ii) $\mathrm{L}_{\mathrm{s}}=62.45-2.12 \mathrm{t}+125.0 \mathrm{~A}+70.97 \mathrm{~F}+3.38 \mathrm{tA}+.80 \mathrm{tF}$

$\mathrm{Si} \quad \mathrm{A}=0$ et $\mathrm{F}=1$

$\mathrm{L}_{\mathrm{sct}}=133.42-1.32 \mathrm{t}$

Si $\quad \mathrm{F} \equiv 0$ et $\mathrm{A}=1$

$\mathrm{L}_{\mathrm{ses}}=187.45+1.26 \mathrm{t}$ 
TABLEAU XVI

Quelques résultats de l'analyse de régression linéaire

pour le secteur des salaires

PATRONAGE DE RÉTRIBUTION +

$$
\begin{aligned}
& \begin{array}{ccccccc}
c_{0} & t & A & F & t A & t F & R^{2}
\end{array} \\
& \mathrm{~L}_{\mathrm{s}}=30.56 \underset{(1.22)}{+.36}+\underset{(40.83)}{+167.42}+\underset{(41.67)}{81.22} \\
& =169.08-9.28 \\
& \text { (3.09) }
\end{aligned}
$$

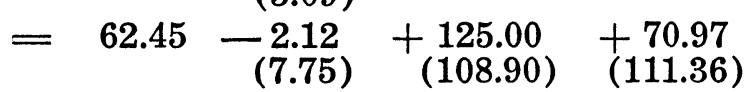

$$
\begin{aligned}
& +11.81+5.60 \\
& \text { (2.96) (3.01) } \\
& .016^{*} \quad(14) \\
& \mathrm{t} \quad \mathrm{X}_{1} \quad \mathrm{X}_{2} \\
& +3.38 \\
& \text { (7.90) }
\end{aligned}
$$

\begin{tabular}{|c|c|c|c|c|c|c|}
\hline & & $\mathbf{X}_{1}$ & $\mathbf{X}_{2}$ & $\mathbf{X}_{8}$ & $X_{4}$ & $\mathrm{X}_{\mathrm{B}}$ \\
\hline CA & $\begin{array}{c}\overline{\mathbf{Q}}=\begin{array}{c}\text { Non-Québec } \\
\text { Québec }\end{array} \\
\text { Ques }\end{array}$ & $\begin{array}{l}0 \\
0\end{array}$ & $\begin{array}{l}0 \\
0\end{array}$ & $\begin{array}{l}0 \\
1 \\
\end{array}$ & $\begin{array}{l}0 \\
0\end{array}$ & $\begin{array}{l}0 \\
0\end{array}$ \\
\hline $\mathrm{CF}$ & $\begin{array}{c}\overline{\mathbf{Q}}=\begin{array}{c}\text { Non-Québec } \\
\text { Québec }\end{array} \\
\text { Q }\end{array}$ & $\begin{array}{l}1 \\
1 \\
\end{array}$ & $\begin{array}{l}0 \\
0 \\
\end{array}$ & $\begin{array}{l}0 \\
1 \\
\end{array}$ & $\begin{array}{l}0 \\
1 \\
\end{array}$ & $\begin{array}{l}0 \\
0\end{array}$ \\
\hline $\begin{array}{l}\text { ETHNICITE } \\
\text { INCONNUE }\end{array}$ & $\overline{\mathbf{Q}}=\begin{array}{c}\text { Non-Québec } \\
\text { Québec }\end{array}$ & $\begin{array}{l}0 \\
0\end{array}$ & $\begin{array}{l}1 \\
1\end{array}$ & $\begin{array}{l}0 \\
1\end{array}$ & $\begin{array}{l}0 \\
0\end{array}$ & $\begin{array}{l}0 \\
1\end{array}$ \\
\hline
\end{tabular}

+ La notation employée est claire sauf pour les 5 variables fictives utilisées dans l'équation (17) pour spécifier la classification croisée des régions et des groupes ethniques. Ces variables ont le sens suivant:

La variable $X_{1}$ s'annule pour tous les postes sauf ceux détenus par des Canadiens français, la variable $X_{2}$ s'annule pour tous les postes sauf ceux détenus par des fonctionnaires dont l'ethnicité est inconnue, la variable $\mathrm{X}_{3}$ s'annule pour tous les postes hors de Québec, la variable $\mathrm{X}_{4}$ s'annule pour tous les postes sauf pour ceux détenus par des Canadiens français à Québec, et la variable $X_{5}$ s'annule enfin pour tous les postes sauf ceux détenus par des fonctionnaires dont l'origine ethnique est inconnue mais qui œuvrent à Québec. Cette structure de variables permettra plus loin de repérer le niveau de salaire prédit pour chacun des six groupes, à partir de la seule équation (17). 
Les fluctuations de ce niveau moyen des salaires sont dues en partie au fait que l'Etat a déjà mis en place les postes supérieurs, au début de la décennie 1800 , et que les postes additionnels établis par la suite, sous la pression de la demande de biens publics par une population accrue, sont surtout des postes subalternes: la moyenne des salaires s'en trouve par conséquent déprimée. ${ }^{184}$ De même que nous avions parlé d'un modèle de type exponentiel pour les pensions, il nous semble qu'il sera utile, dans une prochaine étape, de chercher à insérer explicitement dans un modèle plus complexe, la composition de l'agrégat des postes et plus particulièrement l'importance des phases de la multiplication des subalternes dans l'évolution d'une structure bureaucratique.

Le même phénomène apparaît encore mieux découpé, s'il se peut, quand on s'attache aux niveaux moyens des salaires des deux groupes ethniques dans la même région de Québec. Pour étudier l'effet de l'ethnicité et de la localisation sur le niveau de salaire, il nous a fallu recourir à un assemblage plus complexe de variables fictives. Le tout a été explicité d'ailleurs dans une note attachée au tableau XVI.

A partir des résultats de l'équation (17), il est possible de définir les plateaux correspondant aux divers niveaux de salaire pour les six groupes définis par une ventilation Canadien français, Canadien anglais, ethnicité inconnue et Québec non Québec. Nous avons reproduit et les niveaux moyens observés et les droites de régression correspondant aux deux groupes (Canadiens anglais à Québec, Canadiens français à Québec) qui nous intéressent dans le graphique IV. Ici encore, un modèle plus complexe rendrait un meilleur compte des variations observées. ${ }^{185}$

Quel que soit le caractère rudimentaire de nos calculs, l'équation (17) permet de définir six paliers du genre de ceux

${ }^{184}$ Un coup d'œil rapide au tableau $\mathrm{X}$ situe très bien le démarrage de cet élargissement de la base à l'année 1804 .

185 Par un procédé identique à celui exposé plus haut, on peut dériver six équations de l'équation (17):

$$
\begin{aligned}
& \mathrm{L}_{\mathrm{SCAQ}}=119.74+.31 \mathrm{t} \\
& \mathrm{LsOAQ}=254.08+.31 \mathrm{t} \quad(17 \mathrm{~b}) \\
& \mathrm{L}_{\mathrm{sOFQ}}=127.93+.31 \mathrm{t} \quad \text { (17c) } \\
& \mathrm{L}_{\mathrm{serq}}=102.43+.31 \mathrm{t} \quad \text { (17d) } \\
& \mathrm{L}_{\mathrm{SBI}}=16.24+.31 \mathrm{t} \quad(17 \mathrm{e}) \\
& \mathrm{L}_{\mathrm{BHIQ}}=37.50+.31 \mathrm{t} \text { (17f) }
\end{aligned}
$$

Seules les équations pertinentes, soit $17 \mathrm{~b}$ et $17 \mathrm{~d}$, sont inscrites au graphique IV. 
notés dans le graphique IV. Si nous écartons, pour le moment, les postes pour lesquels nous ne connaissons pas l'origine ethnique des détenteurs (lesquels constituent d'ailleurs les deux derniers paliers), on voit que l'ordre descendant va des anglophones de Québec aux francophones hors de Québec, aux anglophones hors de Québec et aux francophones de Québec.

Ici encore, nos résultats sont donc bien clairs: il y a eu patronage de répartition et de rétribution dans la fonction publique, et ces deux séries de forces ont contribué à décalquer les contours de la fonction publique au tournant du XIX $\mathrm{XIècle}^{\mathrm{e}}$ Nous savons déjà que la probabilité de détenir un poste pour un Canadien français décroît à mesure que les niveaux de salaire s'élèvent, mais s'accroît au cours de la période. Elle s'avère aussi plus élevée pour les postes de Québec. En ce qui a trait aux niveaux de rétribution, il existe un décalage de niveaux entre les groupes ethniques et entre les régions. Bien que nous ayons retenu l'effet de niveau, le graphique III illustre clairement que l'écart s'agrandit entre Canadiens anglais et Canadiens français au cours de la période. Si l'on standardise les données en nous confinant à la région de Québec, l'effet de niveau semble clairement dominer. Cependant, si l'on se reporte au graphique I, on voit que pour Montréal, le renversement des niveaux appelle un modèle d'explication plus complexe.

\section{(5) Propos d'étape}

Ainsi que nous le disions au début de cette section, nous avons voulu tout au plus suggérer un modèle plausible et en donner un test grossier. Malgré sa crudité, notre procédé nous a permis de déceler certaines différences dans les méthodes des patronages de répartition et de rétribution au cours de la période qui nous intéresse. Notons seulement au passage la différence dans le signe de la variable $t$ dans les équations (5) et (9) en tant qu'il affecte la probabilité d'être canadien-français dans le secteur des pensions et des salaires, ainsi que la différence dans l'importance relative de l'effet de pente et de celui de niveau à l'intérieur de l'effet de rétribution pour les pensions et les salaires. Cependant, ici, la nature des sentiers de croissance appelle des modèles plus complexes avant que l'on puisse vraiment trancher la question.

Loin de nous l'idée de regarder ces premières formalisations comme définitives ou complètes. Tout au plus permettent-elles d'étayer provisoirement notre modèle. Dans une prochaine étape, il faudra tenter d'approfondir le problème avec des moyens moins rudimentaires, i.e. en construisant un modèle à plusieurs 
équations qui précise mieux le fonctionnement de l'économie publique bas-canadienne et, par ricochet, les formes du patronage qu'on y trouve.

Entre-temps, nous voudrions examiner de plus près les motivations diverses qui sous-tendent les procédés que nous avons mis à jour, et saisir, dans la dynamique de cette institution qu'est la liste civile, certains éléments de la dynamique de la société dont elle est, en quelque sorte, un produit. C'est à ce travail que nous consacrerons la prochaine section.

$$
\text { (à suivre) }
$$

Carleton University

GILles PaQUeT

Université de Toronto

JEAN-PIERRE WALLOT 